\title{
Lower Hybrid Current Drive for Edge Current Density Modification in DIII-D: Final Status Report
}

\author{
M. E. Fenstermacher \\ Lawrence Livermore National Laboratory \\ Livermore, CA 94550 \\ and \\ M. Porkolab \\ Plasma Fusion Center, MIT \\ Cambridge, MA 02139
}

August 4, 1993

\section{Introduction}

Application of Lower Hybrid (LH) Current Drive (CD) in the DIII-D tokamak has been studied at LLNL, off and on, for several years. The latest effort began in February 1992 in response to a letter from ASDEX (Appendix A) indicating that the $2.45 \mathrm{GHz}, 3 \mathrm{MW}$ system there was available to be used on another device. An initial assessment of the possible uses for such a system on DIII-D was made and documented in September 1992 (see Ref. 1). Multiple meetings with GA personnel and members of the LH community nationwide have occurred since that time. The work continued through the submission of the 1995 Field Work Proposals in March 1993 and was then put on hold due to budget limitations. The purpose of this document is to record the status of the work in such a way that it could fairly easily be restarted at a future date.

This document will take the form of a collection of Appendices giving both background and the latest results from the FY 1993 work, connected by brief descriptive text. Section 2 will describe the final workshop on LHCD in DIII-D held at GA in February 1993. This was an open meeting with attendees from GA, LLNL, MIT and PPPL. Summary documents from the meeting and subsequent papers describing the results will be included in Appendices. Section 3 will describe the status of work on the use of low frequency (2.45 GHZ) LH power and Parametric Decay Instabilities (PDI) for the special case of high dielectric in the edge regions of the DIII-D plasma. This was one of the critical issues 


\section{DISCLAIMER}

This report was prepared as an account of work sponsored by an agency of the United States Government. Neither the United States Government nor any agency thereof, nor any of their employees, make any warranty, express or implied, or assumes any legal liability or responsibility for the accuracy, completeness, or usefulness of any information, apparatus, product, or process disclosed, or represents that its use would not infringe privately owned rights. Reference herein to any specific commercial product, process, or service by trade name, trademark, manufacturer, or otherwise does not necessarily constitute or imply its endorsement, recommendation, or favoring by the United States Government or any agency thereof. The views and opinions of authors expressed herein do not necessarily state or reflect those of the United States Government or any agency thereof. 


\section{DISCLAIMER}

Portions of this document may be illegible in electronic image products. Images are produced from the best available original document. 
identified at the workshop. Other potential issues for LHCD in the DIII-D scenarios are: 1) damping of the waves on fast ions from neutral beam injection , 2) runaway electrons in the low density edge plasma, and 3) the validity of the WKB approximation used in the ray-tracing models in the steep edge density gradients. These are discussed in Sections 4,5, and 6 respectively. A summary of the work status will be given in Section 7.

\section{Final LHCD Workshop at GA, 2/11/93}

Substantive meetings in which LHCD for DIII-D was discussed, were held at GA and LLNL in September and October 1992, informally at the APS meeting in November, at the US/Japan CD workshop at PPPL in December, and at GA in January 1993. The final workshop on the subject was held at GA on February 11, 1993. Representatives from LLNL, MIT and PPPL attended the workshop as well as a large group from GA. The workshop agenda and a memorandum from $T$. Simonen giving the "charges" to the workshop are given in Appendix B.

The studies presented at the workshop focussed on use of LHCD to modify the edge $(0.8<$ rho $<1.0)$ current density. The objective was to produce the current profiles which seem to be required for the advanced confinement regimes in DIIID. In particular, studies were presented in which LHCD was used to drive currents opposite to the plasma current near the edge of the plasma to cancel some fraction of the large edge bootstrap current that develops during the $\mathrm{VH}$ mode. Stability analysis presented at the workshop indicated that control of the edge current density might allow extension of the VH-mode to longer pulses. These LHCD scenarios were subsequently presented at the Rf Power in Plasmas meeting in Boston (April 1993) and the paper from that meeting is included as Appendix C. A detailed directory of cases run for DIII-D with the ACCOME current drive code is given in Appendix D.

A general discussion session was held at the end of the meeting. The conclusions of the workshop, as they related to the "charges," were summarized in a memorandum (see Appendix E). It was concluded that there were attractive advanced tokamak scenarios using LHCD in DIII-D, and that LHCD was supported by a substantial existing database from previous experiments. However, two key issues were raised concerning the counter edge CD scenarios proposed for DIII-D. The first was the density limit, previously seen in other experiments, which simple scaling arguments indicated might be exceeded in the proposed scenarios. The second issue was that of fast electron confinement in the narrow edge plasma regions over which the current was to be driven. It was proposed that attempts be made to get information on these and several other secondary issues from new LH experiments on existing tokamaks. 
Following the workshop several issues were identified for work before the submission of Field Work Proposals (FWP) (see Appendix F). These issues were only partly addressed before the FWP preparation work began. The FWP submitted by LLNL for the 1995 fusion budget included a proposal to install the ASDEX LHCD system on DIII-D. The LHCD proposal section of that FWP is included as Appendix G. The effective response to the proposal was that there were insufficient funds to go ahead with the installation scenario at this time.

\section{Frequency Choice, Density Limits and Paramteric Instabilities}

Choosing the best frequency for the LHCD applications in DIII-D was an issue throughout this work. Given the availability of the ASDEX system at $2.45 \mathrm{GHz}$, it was desireable from the cost standpoint to use this frequency if possible. However, for the edge counter current drive (CCD) applications in typical DIII-D $\mathrm{VH}$-mode plasmas, the local density was projected from simple scaling arguments to be larger than the LH density limit for $2.45 \mathrm{GHz}$ waves. It was also noted however, that the behavior termed the "density limit" occurred in high density experiments in which the local lower hybrid frequency was less that twice the wave frequency, $\mathrm{f} / \mathrm{fLH}<2$. In the edge regions on the outboard side of DIII-D where the waves are damped in the CCD scenarios, f/fLH $>4$ so the density limit due to parametric decay instabilities would be avoided. However, the data from previous experiments were also in a regime for which the local dielectric constant was less than one where the waves were propagating. In the outer edge of the DIII-D plasma in VH-mode, the dielectric constant would be greater than one and little data is available on LH propagation in this regime. This subject was presented at the February workshop and the vugraphs are included in Appendix $\mathrm{H}$. A detailed write-up of the parametric decay instabilities work for high dielectric constant is given in Appendix I.

\section{Fast Ion Damping of LH Waves}

One issue in connection with edge current drive by lower hybrid waves is the competing mechanism of absorption by fast ions in the presence of neutral beam injection. We have briefly examined the question of ion absorption versus electron absorption in the edge region. According to the available data from DIII-D, there is no substantial fast ion population in the edge. Nevertheless, in TPX, Monte-Carlo type simulations are available from the work of Mikkelsen ${ }^{2}$ which indicate that in the edge region fast ions with a mean energy of $\sim 30 \mathrm{keV}$ and with a relative density of $\sim 1 \%$ may be present for $10 \mathrm{MW}$ of $120 \mathrm{keV}$ TFTR 
beams. The dispersion relationship of lower hybrid waves, including damping, may be written in the following form:

$$
\varepsilon(\omega, k)=1+\frac{\omega_{p e}^{2}}{\omega_{c e}^{2}}-\frac{\omega_{p e}^{2}}{\omega^{2}} \frac{k_{11}^{2}}{k^{2}}+i \chi_{e, \mathrm{Im}}+i \chi_{i, \mathrm{Im}}
$$

where the electron damping term (including quasi-linear modification) can be written in the form:

$$
\chi_{e}=\frac{\pi^{1 / 2}}{k^{2} \lambda_{D e}^{2}} \frac{\zeta_{0 e} e^{-\zeta_{0 e}^{2}}}{1+\sqrt{2} \zeta_{0 e}^{3}}
$$

and where the hot ion damping for a maxwellian can be written as:

$$
\chi_{i, \mathrm{Im}}=\frac{\pi^{1 / 2}}{k_{\perp}^{2} \lambda_{D h}^{2}} \zeta_{i h} e^{-\zeta_{i h}^{2}}
$$

Here $a=\zeta_{i h}=\left(\omega / k_{\perp} v_{t h}\right)$ and $v_{t h}$ is the perpendicular mean velocity of the hot ion component. As shown in the ray tracing results (Figs. 1 and 2), typical values of $N_{\perp}$ are in the range $N_{\perp} \cong 60-100$ for $N_{1}$ in the range $4<N_{11}<7$. Corresponding edge plasma parameters are $\mathrm{T}_{\mathrm{e}} \sim 1-1.5 \mathrm{keV}, \mathrm{n}_{\mathrm{e}} \sim(6-7) \times 10^{19} \mathrm{~m}^{-3}$. The damping decrement can be written as:

$$
k_{\mathrm{Im}, \perp}=\frac{\chi_{e, \mathrm{Im}}+\chi_{i, \mathrm{Im}}}{\partial \varepsilon / \partial k_{\perp}}=\frac{\chi_{e, \mathrm{Im}}+\chi_{i, \mathrm{Im}}}{v_{g \perp}(\partial \varepsilon / \partial \omega)}
$$

where $v_{g \perp}=\partial \omega / \partial k_{\perp}$ is the perpendicular component of the group velocity. Clearly electron Landau damping will dominate if $\left|\chi_{e, \mathrm{Im}}\right|>\left|\chi_{i, \mathrm{Im}}\right|$. For typical values of $\mathrm{n}_{\mathrm{b}} \sim 5 \times 10^{17} \mathrm{~m}^{-3}, \mathrm{n}_{\mathrm{e}} \sim 5 \times 10^{19} \mathrm{~m}_{-}{ }^{3}, \mathrm{n}_{\mathrm{b}} / \mathrm{n}_{\mathrm{e}} \sim 10^{-2}, \mathrm{~T}_{\perp \mathrm{h}} \sim 30 \mathrm{keV}, \mathrm{T}_{\mathrm{e}} \sim 1$ $\mathrm{keV}$ we find that $\chi_{\mathrm{e}_{\mathrm{I}} \mathrm{Im}} \sim(1-2) \times 10^{-2}, \chi_{\mathrm{i}, \mathrm{Im}} \sim 10^{-4}$ an thus $\chi_{\mathrm{i}, \mathrm{Im}} / \chi_{\mathrm{e}, \mathrm{Im}} \sim<10^{-2}$. Therefore absorption on hot ions is negligible as compared with absorption on electrons. We have explored parameters in the range $\zeta \sim 0.88-0.95$, and these conclusions remain unchanged. It can be seen that $n_{b} / n_{e} \sim 0.1$ would be necessary near the edge to produce substantial absorption by ions. Thus, current drive remains viable at the edge. We have also looked at a more accurate treatment of ions, namely considering a slowing down distribution of ions. The results remain essentially the same. 


\section{Electron Runaway Conditions}

A concern was raised during the workshop that the residual electric fields in the edge of the $\mathrm{VH}$-mode plasmas might produce runaway electrons from the fast electron tail generated by the LH waves. The runaway electric field condition can be written as:

$$
E_{r} \geq 33 \frac{n_{e 20}(\Psi) \sqrt{T_{e}(\mathrm{keV})(\Psi)}}{E_{\text {tail }}^{3 / 2}(\mathrm{keV})}
$$

where Etail is the energy of the LH generated fast electrons. For the scenarios described above, Etail $\sim 25 \mathrm{keV}$ (at high $\mathrm{N}_{\|}>5$ ), $\mathrm{n}_{\mathrm{e} 20} \sim 0.5$ and $\mathrm{T}_{\mathrm{e}} \sim 0.5 \mathrm{keV}$ so that the condition for runaways is $\mathrm{E}_{\mathrm{r}}>0.093 \mathrm{~V} / \mathrm{m}$. In the flattop phase of the discharge, during which the LHCD would be used, the loop voltage is typically Vloop $<0.2 \mathrm{~V}$, so that the electric field is $\mathrm{E}<0.02 \mathrm{~V} / \mathrm{m}$. Therefore runaway electrons should not be a problem for the high $\mathrm{N}_{\|}$(low phase velocity) scenarios considered here. This has been confirmed in Fokker-Planck modeling as described in Appendix C.

\section{Validity of Ray Tracing Approximations}

The WKB approximation used in the ray-tracing model is valid if the following condition is satisfied:

$$
\frac{\omega^{2}}{c^{2} k_{\perp}^{2}}\left(\frac{d k_{\perp}}{d x}\right) \frac{c^{2}}{\omega^{2}} \equiv \frac{1}{N_{\perp}^{2}} \frac{d N_{\perp}}{d x} \frac{c}{\omega}<1
$$

The perpendicular index of refraction can be evaluated as:

$$
N_{\perp} \cong N_{\|}\left(\frac{\omega_{c e}}{\omega}\right)\left(1-\frac{1}{2} \frac{\omega_{c e}^{2}}{\omega_{p e}^{2}}\right)
$$

if $\omega_{c e}^{2} \ll<\omega_{p e}^{2}$ so that the validity condition can be written as:

$$
\frac{c}{\omega_{c e}} \frac{1}{N_{\|}}\left(\frac{1}{2} \frac{\omega_{c e}^{2}}{\omega_{p e}^{2}} \frac{1}{L_{n}}\right)\left(1+\frac{\omega_{c e}^{2}}{\omega_{p e}^{2}}\right)<1
$$

where $L_{n}$ is the density gradient scale length. In the edge region on the outboard side of the DIII-D VH-mode plasmas considered here, $\omega_{p e}^{2} / \omega_{c e}^{2}=4, \omega_{c e} \sim 2 \pi 40$ 
$\mathrm{GHz}$, and the scenarios presented above use $\mathrm{N}_{\| \sim} 5$ so the validity condition becomes, $0.004 \mathrm{~cm} / \mathrm{L}_{\mathrm{n}}<1$. This is well satisfied for the measured scale length, $L_{\mathrm{n}} \sim 0.1 \mathrm{~cm}$, in the edge of these plasmas.

\section{Summary}

The status of the LLNL/MIT/GA work on LHCD in DIII-D as of April, 1993 has been reviewed. Attractive $\mathrm{LH}$ counter-current drive scenarios in which the $\mathrm{LH}$ current partially cancels the excessive bootstrap current at the edge of the $\mathrm{VH}$ mode plasma have been found using $2.45 \mathrm{GHz}$ waves. The power level of the ASDEX LH system ( $3 \mathrm{MW}$ ) was sufficient in the modeling to drive approximately $100 \mathrm{kA}$ of counter current. This was marginally sufficient for cancellation of the excessive bootstrap current. Two important issues for these scenarios were raised during this work: 1 ) the fact that the experiments would be in a new dielectric regime for LHCD and that the onset of parametric decay instabilities (the LH "density limit") has not been tested in this regime, and 2) that the particle confinement of the fast current carrying electrons was important since the scenarios require very localized current generation. Modeling work on the density limit issue, showing that this regime should not produce substantial depletion of the pump wave based on PDI theory, is reported here. Characterization of the fast electron particle confinement and tests of the PDI predictions must be obtained from existing experiments. Overall, the idea of using LHCD, in a complementary way with the other core CD systems on DIII-D, to modify the current density profile at the edge of the advanced performance plasmas, and thereby extend the pulse length, remains attractive and should be pursued when funds become available. 


\section{References}

1. M.E. Fenstermacher and M. Porkolab, "Initial Assessment of Lower Hybrid Current Drive in DIII-D," Lawrence Livermore National Laboratory report UCRL-ID-111838, September 1992.

2. M. Porkolab, D. Mikkelsen, et. al., Chapter 7 in the TPX Physics Design Description, March 29, 1993, Report 93-930319-PPPL/GNeilson-01.

3. M. Mayberry, M. Porkolab, et. al., "Frequency Scaling of the Lower-HybridCurrent-Drive Density Limit in Tokamak Plasmas," Phys. Rev. Lett., 55, 19Aug1985, pp.829-32.

4. M. Porkolab, "Parametric Instabilities Due to Lower-Hybrid Radio Frequency Heating of Tokamak Plasmas," Phys. Fluids 20, 2058, 1977.

5. Y. Takase and M. Porkolab, "Parametric Excitation of Ion-Sound Quasimodes During Lower-Hybrid Heating Experiments in Tokamaks," Phys Fluids $\underline{\mathbf{2 6}}$, 2992, 1983.

\section{List of Appendices}

Appendix A

Appendix B

Appendix C

Appendix D

Appendix E

Appendix F

Appendix G

Appendix $\mathrm{H}$
Letter from Prof. K. Lackner, ASDEX indicating the availability of the $2.45 \mathrm{GHZ}, 3 \mathrm{MW}$ LHCD system.

Agenda of the final workshop on LHCD in DIII-D held at GA, 2/11/93 and Memorandum giving the "charges" to the workshop.

Paper on LHCD in DIII-D given at the Rf Power in Plasmas meeting, Boston, April 1993.

Summary of the general discussion session of the 2/11/93 workshop.

Directory of ACCOME cases for LHCD in DIII-D.

Memooandum on tasks to be completed before FWP's.

Section of the LLNL FWP on LHCD in DIII-D.

Viewgraphs on frequency choice and PDI presented at the 2/11/93 workshop. 
Appendix I Memorandum on frequency choice and details of PDI calculations. 
Appendix A Letter from K. Lackner, ASDEX 


\section{MAX-PLANCK-INSTITUT FÜR PLASMAPHYSIK}

TOKAMAKPHYSIK Prof. Karl Eackner

Dr. P.H. Rutherford

University of Princeton

Plasma Physics Laboratory

James Forrestal Campus

P.O.Box 451

Princeton, N.J. 08545

USA



PAUL H. RUTHERFOND
D-8046 GARCHING

Boltzmannstr. 2

Tel.: 089/3299-2204

Telefax 089/3299-2580

May 28, 1991

Dear Paul,
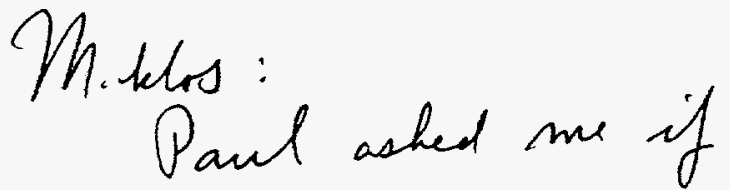

we

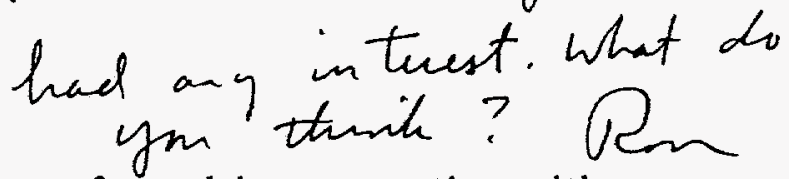

The $2.45 \mathrm{GHz}$ lower hybrid experiments, which were performed in cooperation with ENEA and PPPL, have been terminated together with ASDEX in August last year. We have now to decide what to do with the lower hybrid system, parts of which are the property of the contributing laboratories.

Specifically these are:

from ENEA: 3 klystrons with magnets

6 sockettanks

6 transmissionlines

grill antenna and feeding structure

miscelaneous waveguide components

from PPPL: 3 klystrons with magnets

3 vacuum windows.

Contrary to earlier ideas, IPP has currently no plan to use this system in the ASDEX Upgrade experiment. However, since this is a well-working and reliable system, it would be a pity to disassemble it prior to having decided on the form of its further use. We therefore propose to leave it in its present state for another year, say until July 1992, provided there are no other requests from your side concerning this system or parts of it.

Best regards

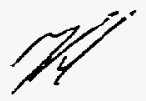

Karl Lackner 


\title{
Appendix B 1. Agenda of LHCD Workshop
}

\author{
Informal Workshop on \\ Lower Hybrid Current Drive in DIII-D \\ General Atomics, San Diego CA \\ February 11, 1993
}

Agenda

9:30 Fenstermacher Welcome, Comments on the Agenda

9:35 Freeman DIII-D Five Year Plan Scenarios

$20 \mathrm{~min}$

9:50 Fenstermacher LLNL View of LHCD Mission on DIII-D

$30 \mathrm{~min}$

10:10 Nevins

TPX Relevance of Edge Counter CD

$20 \mathrm{~min}$

10:30 Taylor(leader)

Discussion of possible LHCD

$10 \mathrm{~min}$

Mission on DIII-D

11:10 Break

11:20 Greenfield VH-mode Stability and Transport Considerations $20 \mathrm{~min}$

Noon Lunch

1:00 Porkolab

$30 \mathrm{~min}$

LHCD Density Limits and Frequency Choices

1:50 Fenstermacher

LHCD Scenarios in VH-mode-like DIII-D Plasmas $30 \mathrm{~min}$

2:20 Harvey

$10 \mathrm{~min}$

Fokker-Planck Modeling of Edge LHCD in DIII-D

2:50 Lin-Liu

$20 \mathrm{~min}$

ECH Applications for Advanced Tokamak Modes 


$\begin{array}{lll}\text { 3:00 } & \begin{array}{l}\text { Bernabei } \\ 30 \mathrm{~min}\end{array} & \text { Comments } \\ 3: 20 & \text { Break } & \\ 3: 30 & \text { All } & \text { Discussion of ASDEX-System Engineering Status } \\ \text { 4:00 } & \text { Nevins(leader) } & \text { General Discussion } \\ 5: 00 & \text { Adjourn } & \end{array}$




\section{Appendix B 2. Charges to the Workshop}

GEMERAL ATOMICS

\section{Memorandum}

Date: $\quad$ February 10, 1993

From: $\quad$ Thomas C. Simonen

To: $\quad$ Distribution

Subject: Questions about Lower Hybrid Current Drive on DIII-D

The following are questions you could address at Thursday's Lower Hybrid meeting to help me understand the role of Lower Hybrid on DIII-D.

1. Are there attractive AT (advanced tokamak) DIII-D LH scenarios?

2. Are the AT scenarios base on an established LH data base?

3. How much of the AT physics can be explored with inductive edge current drive?

4. Could the proposed LH scenarios be tested on C-mod or PBX-M?

5. Given the many LH experiments worldwide and the pioneering work on ECH at DIII-D why would DIII-D de-emphasize ECH in favor of LH?

\section{Distribution:}

R. Freeman, V. Chan, R. Callis, C. Moeller, T. Taylor, P. Politzer, A. Turnbull, S.C. Chiu, R. Harvey, R. Prater, R. Pinsker, T. Luce, C. Forest, Y. Lin-Liu, C. Petty, H. St. John, W. Nevins (LLNL), M. Porkolab (MIT). 


\section{Appendix C LHCD Paper from the Rf Power in Plasmas Meeting, Boston, 1993}




\author{
Lower Hybrid Counter Current Drive for Edge Current Density \\ Modification in DIII- $\mathrm{D}^{*}$ \\ M.E. Fenstermacher and W.M. Nevins \\ Lawrence Livermore National Laboratory, Livermore, CA 94550 \\ M. Porkolab and P.T. Bonoli \\ Plasma Fusion Center, MIT, Cambridge, MA 02139 \\ R.W. Harvey \\ General Atomics, San Diego, CA 92186
}

\begin{abstract}
Each of the Advanced Tokamak operating modes in DIII-D is thought to have a distinctive current density profile. So far these modes have only been achieved transiently through experiments which ramp the plasma current and shape. Extension of these modes to steady state requires non-inductive current profile control, e.g. with lower hybrid current drive (LHCD). Calculations of LHCD have been done for DIII-D using the ACCOME ${ }^{1}$ and CQL3D ${ }^{2}$ codes, showing that counter driven current at the plasma edge can cancel some of the undesireable edge bootstrap current and potentially extend the VH-mode. Results will be presented for scenarios using $2.45 \mathrm{GHz} \mathrm{LH}$ waves launched from both the midplane and off-axis ports. The sensitivity of the results to injected power, $n_{e}$ and $T_{e}$, and launched wave spectrum will also be shown.
\end{abstract}

\title{
INTRODUCTION
}

Recent experiments in DIII-D have focussed on four modes of plasma operation which have produced either enhanced confinement and/or high beta stablity under transient conditions. Known as the VH-mode, the high $l_{i} \mathrm{H}$-mode, the second stable core mode, and the high $\epsilon \beta_{p}$ mode, each is thought to have a distinct current density profile. In particular, large bootstrap current density at the plasma edge builds up during the VH-mode and may need to be cancelled by a non-inductive counter current drive system to extend this mode to steady state.

The existing current drive program on DIII-D, including Neutral Beam (NB) injection, Electron Cyclotron (EC) harmonic waves, and Ion Cyclotron (ICRF) Fäst Waves (FW), allows the current density profile in the plasma core (near the plasma axis and out to about $2 / 3$ of the plasma radius) to be modified. However, none of these systems has shown a high enough current drive efficiency to affect the current density profile near the plasma edge where the temperature is comparatively low.

LHCD has comparatively high efficiency in this region, the waves can be strongly damped there with proper choice of launched spectrum, and there exist simulation codes that have been benchmarked to previous experimental results. Also, the power sources and other components of the necessary LHCD system for this DIII-D application are readily available and have been proven on previous experiments at ASDEX and PLT. This paper presents ray-tracing, power absorption and counter current drive calculations for LHCD in DIII-D VH-mode-like plasmas.

* Work performed for the U. S. DoF, by T.I:NI: under contract. W-7405-FNG-48 and by GA under contrast DF-AC03-89FR5.3277. 


\section{VH-MODE MODEL PLASMA}

The plasma conditions assumed for the LH ray-tracing and CD calculations are those of DIII-D shot 75121 at $2540 \mathrm{~ms}$. This was a double null (vertically symmetric) plasma with $B_{T}=2.13 \mathrm{~T}, I_{p}=1.63 \mathrm{MA}, R_{o}=1.67 \mathrm{~m}, a=0.62 \mathrm{~m}, \kappa_{x}=2.1, \delta_{x}=0.89$, and $Z_{\text {eff }}=2.0$. The shot progressed from L-mode to the H-mode and finally into the VH-mode at approximately $2400 \mathrm{~ms}$. Analysis of the VH-mode phase has shown that the edge bootstrap current density continually increased throughout the period until it terminated at approximately $2600 \mathrm{~ms}$. Plasma profiles for the fully developed VH-mode at $2540 \mathrm{~ms}$ are shown in Fig. 1. Also given is the profile of the bootstrap current density, showing a maximum of $0.42 \mathrm{~A} / \mathrm{cm}^{2}$ at $\rho=0.9$. The integrated bootstrap current in the region $0.75<\rho<1.0$ was estimated to be $130 \mathrm{kA}$. It is not clear how much of this edge bootstrap current must be cancelled to extend the VH-mode. The objective of the LHCD calculations was to determine whether counter current could be driven in this region and to calculate the $C D$ efficiency there.

\section{CQL3D RESULTS}

In addition to the efficiency and radial location of current drive for a given input power spectrum, an important question is whether the LH driven electron tail leads to appreciable Ohmic conductivity enhancement ("hot conductivity") which for the counter-LHCD case reduces the overall efficiency of the edge current control. The CQL3D Fokker-Planck code ${ }^{2}$ is a comprehensive 2D-in-velocity-space, 1D-in-radius computational model which addresses these problems. It incorporates a $\mathrm{LH}$ ray tracing code, includes self-consistent damping of the energy flowing along the rays, the effects of trapping, and the toroidal electric field. Here it is used with the noncircular equilibria of the shot described above.

Fig. 2 shows current profiles obtained with CQL3D, using the experimental $0.1 \mathrm{~V}$ loop-voltage and $2 \mathrm{MW}$ of LH power incident from the plasma periphery on the outer equatorial plane, with $N_{\|}$from 4.0 to 6.0. The net current density was substantially flattened out near the plasma edge by the LHCD. Damping in these cases is essentially single pass.

Varying $N_{\|}$from 3.5 to 7.0 (central value) gave driven rf currents from 160 . to 70. $\mathrm{kA}$, respectively, peaked in the range of radial $\rho$ values from 0.75 to 0.975 . The optimal situation for cancelling the bootstrap current near the plasma edge was with the $N_{\|}$spectrum from 4.0 to 6.0 , which gave $100 \mathrm{kA}$ rf current peaked at $\rho=0.85$. For the values of loop voltage obtained in the experiment $\left(V_{\phi}=0.1-0.2\right.$ volts) there was no appreciable synergy between the LHCD and the toroidal electric field. That is, the Ohmic and rf currents simply added.

Again using CQL3D, a series of code runs were performed to evaluate possiblities for increasing the LHCD efficiency using combinations of two injected spectra. The higher $N_{\|}$spectrum can set the radial position of peak damping near the plasma edge, and the lower spectrum will increase the tail at that location. ${ }^{3}$ Combining one-third power in $N_{\|}=3-4$ with two-thirds power in $N_{\|}=4-6$ for a total of $2 \mathrm{MW}$ input power, gave $120 \mathrm{kA}$ peaked at $\rho=0.85$, to be compared with $100 \mathrm{kA}$ for the pure $N_{\|}=4-6$ spectra. Thus efficiency is increased by 20 percent. Using a half-and-half split of the power in the above two spectra, the total current was $130 \mathrm{kA}$, still peaked at $\rho=0.85$, but the radial width of the current generation became greater than the extent of the bootstrap 
hump. If the spectra do not touch each other in $N_{\|}$, the damping locations decouple. Thus a compound spectra (two independent antenna) capability adds flexibility to the experiment, although the useful efficiency increase is only of order 20-30 percent.

\section{ACCOME RESULTS}

The ACCOME/SELENE code package ${ }^{1}$ calculates a self-consistent $2 \mathrm{D}$ free boundary plasma equilibrium and non-inductively driven currents from NB, ICRF and LH injection and bootstrap currents. The LH ray-tracing and current drive package ${ }^{4}$ uses a 1-D Fokker-Planck equation with an effective perpendicular temperature. Quasi-linear damping is calculated and the driven current is obtained from the adjoint formulation of Karney and Fisch. ${ }^{5}$

The plasma profiles are input to ACCOME in terms of normalized poloidal flux, $\psi$ as $X(\psi)=\left(X_{o}-X_{a}\right)\left(1-\psi^{\beta_{x}}\right)^{\alpha_{x}}+X_{a}$ where $X=\left(n_{e}, T_{e}\right.$, or $\left.T_{i}\right), X_{o}$ and $X_{a}$ are the central and separatrix values. The VH-mode data from shot 75121 were fit with these functions using $X_{o}, X_{a}, \beta_{x}$, and $\alpha_{x}$ as fitting variables. The weighting function emphasized the outer data more strongly than the core so that the edge bootstrap current density calculated by ACCOME would accurately reflect the experiment.

Rays were launched from the plasma separatrix adjacent to the off axis DIII-D port above the midplane. This was done in an attempt to achieve the maximum toroidal upshift of the launched $N_{\|}$spectrum as the LH wave cone propagated into the plasma. This has the desireable effect of reducing the required $N_{\|}$of the spectrum launched at the antenna which simplifies the antenna design.

For the baseline calculations, the absorbed power in the plasma was $P_{l h}=3.0$ MW, two Gaussian peaks were used in the spectrum with central refractive indices, $N_{\| o}=4.5$ and 6.0 , and $1 / e$ width, $\Delta N_{\|}=0.33$. The counter LH current, $I_{L H}=-86$ $\mathrm{kA}$, was driven in the outer region, $0.75<\psi<0.95$. The efficiency was, $\eta_{l h}=-0.028$ $\mathrm{A} / \mathrm{W}$.

Given the $N_{\|}$upshift due to the off-midplane injection, the comparable case with CQL3D has $N_{\|}=5-7$ and an efficiency, $\eta_{l h}=0.038$, about $35 \%$ higher than the result above. This enhancement is likely due to both the broad $N_{\|}$spectrum used (reaching higher $T_{e}$ ) and to 2-D velocity space effects not adequately modeled in ACCOME with the assumption of $T_{e \perp}=2.5 T_{e, t h}$. Benchmarking of the codes continues.

Results of an ACCOME scan of absorbed power using the baseline spectrum values are given in Fig. 3, showing a constant current drive efficiency. Also, in anticipation of density control with the DIII-D divertor cryopump, cases for higher plasma temperature and lower density (constant pressure) were done for baseline profile shape parameters, $P_{l h}=3 \mathrm{MW}$, and $N_{\|}$adjusted to keep the counter LH current at approximately the baseline radial location. Results are also shown in Fig. 3. The current density profiles, for the case with $T_{e}=9.2 \mathrm{keV}$, are shown in Fig. 4 .

\section{SUMMARY}

It has been shown that LH waves, with a spectrum launched in the counter current direction can modify the edge current density profile in DIII-D VH-mode plasmas. LH counter current drive efficiency in the region of undesireable bootstrap current, $0.8<\rho<1.0$, was in the range $0.028<\eta<0.065$ for the calculations done with 
the present VH-mode plasma. The higher values were obtained with the full FokkerPlanck treatment and a compound wave spectrum launched from the midplane port. Scans at constant plasma pressure showed a factor of 2.2 efficiency increase when the temperature (density) increased (decreased) by $50 \%$.

\section{REFERENCES}

1 K. Tani, et. al., J. Comput. Phys. 98, 332, (1992).

2 R.W. Harvey and M.G. McCoy, to be published in Proc. of IAEA TCM on Advances in Simulation \& Modeling of Thermonuclear Plasmas, Montreal, 1992.

3 F. X. Söldner et. al., in Proc. of 17th European Conf. on Contr. Fusion and Plasma Physics, Amsterdam (1990).

4 P.T. Bonoli and R.C. Englade, PF, 29, 2937, 1986.

5 C.F.F. Karney and N.J. Fisch, PF, 28, 116, 1985.

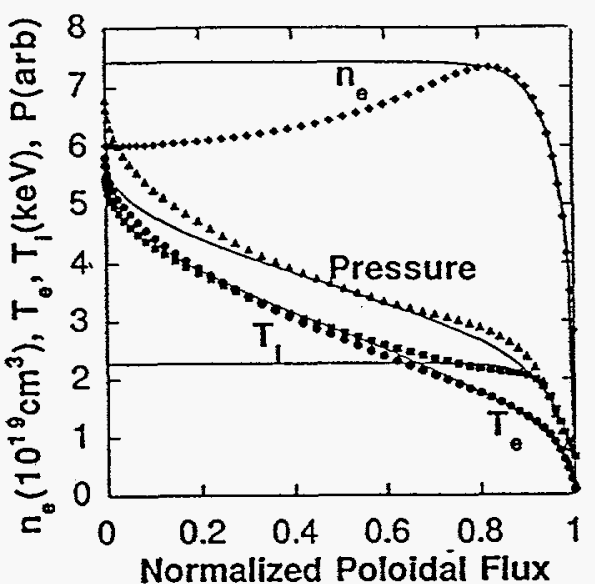

Fig. 1 Profiles of $n_{e}, T_{e}, T_{i}$, and pressure (points) and fits used in ACCOME (lines).

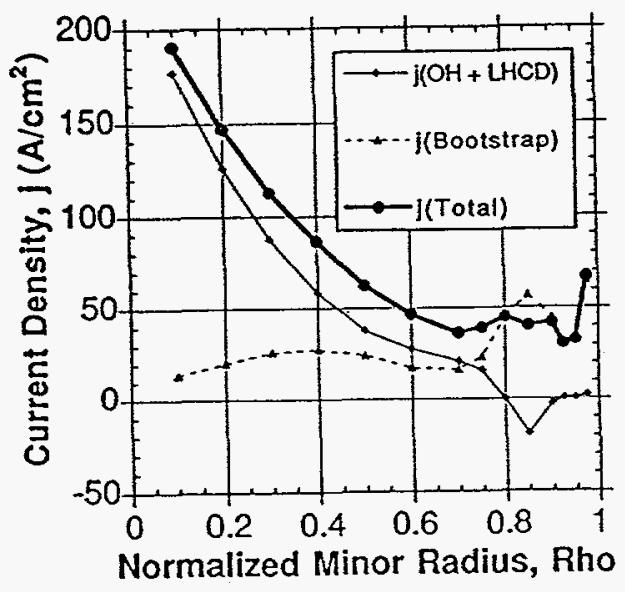

Fig. 2 Current density profiles from CQL3D for $N_{\|}=4-6$, $T_{\text {eo }}=6.1 \mathrm{keV}$, and $P_{l h}=2 \mathrm{MW}$.

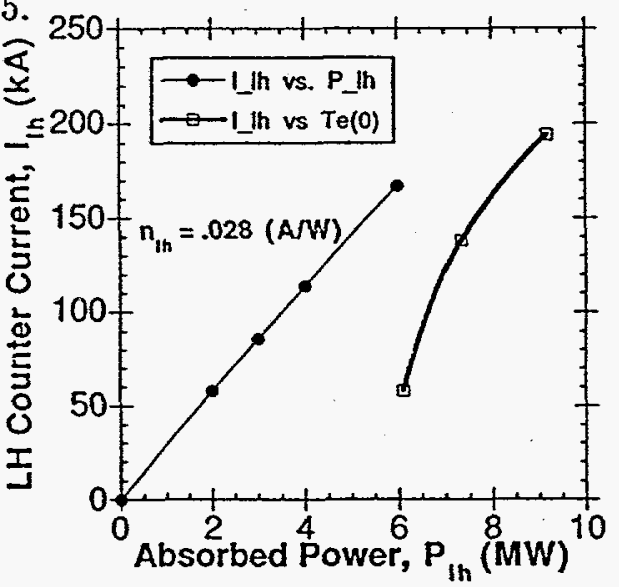

Electron Temperature, $\mathrm{T}_{\mathrm{e} 0}(\mathrm{keV})$

Fig. 3 Lower hybrid counter current vs. $P_{l h}$ and $T_{e}\left(1 / n_{e}\right)$ from ACCOME sensitivity scans.

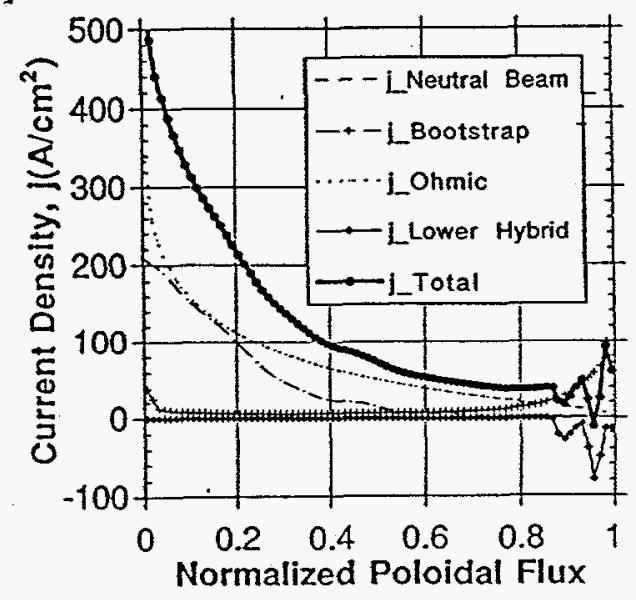

Fig. 4 Current density profiles from ACCOME for $N_{\mid \xi}=3.7,5.4$, $T_{e o}=9.2 \mathrm{keV}$, and $P_{l h}=3 \mathrm{MW}$. 


\section{Appendix D Summary of the Workshop}

\section{Memorandum}

February 1 2, 1993

To: $\quad$ T.C. Simonen, K.I. Thomassen

From: W.M. Nevins

Re: $\quad$ Summary of 2/11/93 Informal Workshop on Lower Hybrid Current Drive on DIII-D

The summary of this meeting is cast as answers to the five questions posed in Simonen's memo of $2 / 11 / 93$, and three further questions from R. Freeman directed at hardware requirements.

\section{Simonen's Questions}

\section{Are there attrative AT (Advanced Tokamak) DIII-D LH scenarios?}

There was general agreement on the existance of attractive AT scenarios for DIIID using lower hybrid power. In particular, we identified scenarios involving tailoring the current profile at the plasma edge with lower hybrid current drive .

In this workshop we focussed on scenarios that addressed goals of the DIII-D five year plan. Our understanding (from R. Freeman's presentation) is that meeting the five-year plan goals will require operation at full current (2MA) and high den.sity $\left(<\mathrm{n}_{\mathrm{e}}>>0.7 \times 10^{20} \mathrm{~m}^{-3}\right)$. At these high densities (and DIII-D's magnetic field of $2 \mathrm{~T}$ ) a combination of the accessibility and quasi-linear damping constraints limit lower hybrid waves to the outer $1 / 3$ of the plasma minor radius. This conclusion holds for all $\mathrm{LH}$ frequencies considered $(2.45$ and $3.7 \mathrm{GHz})$, and limited our attention to scenarios involving edge current drive. Edge current drive requires a high $N_{\|}$launcher, and therefor favors the lower frequency since it is easier to fabricate a high- $\mathrm{N} \|$ launcher for lower frequencies

Note that it would be possible to employ lower hybrid power to modify the current profile in the core of the DIII-D plasma at lower density. 


\section{Are the AT scenarios based on an established LH data base?}

While there is a substantial data base for lower hybrid current drive, the proposed DIII-D scenarios raise several new issues.

The most significant issue is the density limit for lower hybrid current drive (which is thought to be related to parametric decay instabilities). Scaling from previously observed LH density limits would lead us to expect a limit of about $0.4 \times 10^{20} \mathrm{~m}^{-3}$ at $2.45 \mathrm{GHz}$ on DIII-D (which would be too low). However, these DIII-D scenarios are in a regime where $\omega_{p e}^{2} / \omega_{c e}^{2}=4$, while previous LH experiments have generally operated in the regime $\omega_{p e}^{2} / \omega_{c e}^{2}<1$. Porkolab presented calculations indicating that the density limit would be higher in the proposed scenarios because $\omega_{p e}^{2} / \omega_{c e}^{2}>1$. While these calculations where convincing, an experimental demonstration is necessary before spending the money required to install a $2.45 \mathrm{GHz}$ system on DIII-D. Such data may already exist from experiments on ASDEX (and possibly JT-60U). If such data is not available, it could he obtained from experiments on Versator.

A second issue is achieving sufficient confinement of the LH-tail electrons at the plasma edge. This issue could be addressed in experiments on PBX-M. However, the tail electron energy in the proposed DIII-D LH scenarios is rather low (less than $30 \mathrm{keV}$ ), so the slowing-down time is only $1 \mathrm{~ms}$ (hence, only $1 \mathrm{~ms}$ of radial confinement at the plasma edge is required for $\mathrm{j}$-profile control). If data on the confinement of $\sim 30 \mathrm{keV}$ electrons at the plasma edge proves unobtainable from PBX-M, I would judge it an acceptatable risk to address this issue with the proposed DII-D lower hybrid system.

Other issues raised included an absence of data on "backward" current drive with LH power, the effect of inductive electric fields at the plasma edge, and the need for careful modeling of LH wave propagation in the presence of the very steep density gradient at the plasma edge in VH-mode.

\section{How much of thc AT physics can be explored with inductive edge current drive?}

There was general agreement that inductive edge current drive could provide control of the edge current density for times of the order of the edge skin time. Given our current understanding of $\mathrm{VH}$-mode, we may estimate this time by the time over which the VH-mode persists without edge current control (ie. about 0.5 s). Hence, edge inductive current drive could be expected to extend VH-mode for an additional 0.5 seconds. On a longer time scale the inductive current will penetrate beyond the plasma edge and begin to modify the current profile in the plasma core. Hence, edge lower hybrid current drive will be required to control the edge current density for durations of 2-10 seconds. 


\section{Could the proposed scenarios be testcd on C-mod or PBX-M?}

Clearly, these scenarios could be tested on any diverted tokamak given enough money. However, if we limit our attention to equipment on hand, then C-Mod must be ruled out (no LH system). The scenarios could be tested on PBX-M. A new "nose piece" would be required for the PBX-M LH launcher, so these tests could not be performed during the next PBX-M run period.

In addition to PBX-M, there are LH systems on JET, Tore-Supra, and JT-60U. However, all of these systeme are presently designed to launch low $\mathrm{N}_{\|}$(generally $N_{\|} \approx 2$ ), while the edge current profile control requires high $N_{\|}\left(N_{\|} \approx 4-8\right)$, so substantial modifications to the launcher would be required on these experiments.

5. Given the many LH experiments worldwide and the pioneering work on ECH at DIII-D, why would DIII-D de-emphasize ECH in favor of LH?

We found that the proposed LH system complimented the DIII-D ECH system in that it would drive current at the edge, where EC current drive is ineffective. Calculations presented by Lin-Liu showed that $8 \mathrm{MW}$ of ECH power would drive about half as much current as $3 \mathrm{MW}$ of LH power in the edge of a DIII-D VH-mode plasma (ie. the electron current drive efficiency is lower by a factor of 5). It was recognized that the proposed LH system might compete with the $\mathrm{ECH}$ system for limited resources. However, we concluded that our technical workshop should not address funding issues.

\section{Freeman's Questions}

6. Define hardware requiremets for desired applications (frequency, pulse length, power, $\mathrm{N}_{\|}$)

$$
\begin{array}{ll}
\mathrm{f}=2.45 \mathrm{GH} & (3.6 \mathrm{GHz} \text { may also be possible } \\
\mathrm{N}_{\|}=4-8 & \text { (require phase control to vary } \left.\mathrm{N}_{\|}\right) \\
\tau_{\text {pulse }}=10 \mathrm{sec} & \text { (at a minimum, initially } 1 \mathrm{~s}, \text { upgradable to more than } 2 \mathrm{~s} \text { ) } \\
\mathrm{P} \geq 3 \mathrm{MW} & \text { (5 MW needed to provide prudent margin) }
\end{array}
$$

7. Does the ASDEX sy.stem satisify any of the hardware requirements?

It satisfies some. However we would need:

1. A new launcher (ASDEX launcher desighed for low $\mathrm{N}_{\|}$) 
2. A longer pulse length (ASDEX system is $1 \mathrm{sec}$. pulse). This might be an upgrade?

3. More power (ASDEX tubes will only deliver $2 \mathrm{MW}$ beyond $1 \mathrm{sec}$.)

8. What supporting experiments on DIII-D or tokamak devices with LHCD systems are needed to further assess the viability of lower hybrid for Advanced Tokamak studies?

1. Density limit (Versator? Check ASDEX data at low B).

2. Edge $\mathrm{CD} /$ tail electron confinement (PBX-M)

3. Backward CD (PBX-M, ASDEX, others?)

4. Characterize scrape-off-layer plasma in DIII-D at proposed launcher location. 


\section{Appendix E Directory of ACCOME Cases}

The DIII-D LHCD cases run with the ACCOME code are stored under the CFS system at NERSC in the directories 1030347/accomeldIII-d (original Devoto/Porkolab cases) and 1030347/accomeu/diii-d. For the latter (cases described in Ref. 1 and this report), subdirectories are of the form $d 3 d n n$ where $n n$ is a two digit integer (so far in the range $01-11$ ). Files for individual runs are of the form d3dnnab.xxx where $n n$ corresponds to the subdirectory numbering, $a b$ are alphabetic characters (if two characters are used, then $a=" n$ " indicates that the run was made with the new up/down asymmetric version of ACCOME), and $x x x=$ "inp", "bat", "nqs", "out", "plt", "eqb", "trm", "log" for input, interactive script, nqs batch script, output, plot, equilibrium data, terminal text, and batch log files respectively.

The LH and plasma physics attributes for the subdirectories are as follows:

d3d01 - $2.45 \mathrm{GHz}$, "Low" n0=.375, T0=4.7, Second Stable Everywhere scenarios d3d02 - $2.45 \mathrm{GHz}$, "Low" $\mathrm{n} 0=.375, \mathrm{~T} 0=4.7, \mathrm{VH}$-mode scenarios

$d 3 d 03$ - $2.45 \mathrm{GHz}$, "High" n0=0.5, $\mathrm{T} 0=10$, Second Stable Core scenarios

d3d04 - $2.45 \mathrm{GHz}$, "High" n0=0.5, T0=10, Negative edge current for VH- or High $\mathrm{l}_{\mathrm{i}} \mathrm{-H}$ mode scenarios

d3d05 - 4.6 GHz, "High" n0=0.5, T0=10, Second Stable Core scenarios

New up/down asymmetric code

6dd06n - $3.7 \mathrm{GHz}$, "Low" n0, T0 from VH-mode shot, VH-mode scenarios

Analysis with data from Shot 75121 at $2540 \mathrm{~ms}$

$d 3 d 07 n-2.45 \mathrm{GHz}$, Development of baseline case $d 3 d 07 n i$ with HazeltineHinton bootstrap model

$d 3 d 08 n-2.45 \mathrm{GHz}$, NQS batch for $\mathrm{N}_{11}$ sca, (runs $a-f$ )

$d 3 d 09 n-2.45 \mathrm{GHz}, \mathrm{NQS}$ batch for $\mathrm{T}_{\mathrm{e}}$ and $1 / \mathrm{n}_{\mathrm{e}}$ scan, (runs $a-f$ )

$d 3 d 10 n-2.45 \mathrm{GHz}$, NQS batch for Power scan, (runs $a-g$ )

Physics bug fixed in XTIK affecting negative current

$d 3 d 08 n-2.45 \mathrm{GHz}$, NQS batch for $\mathrm{N}_{11}$ scan, (runs $g-i$ )

$d 3 d 09 n-2.45 \mathrm{GHz}$, NQS batch for $\mathrm{T}_{\mathrm{e}}$ and $1 / \mathrm{n}_{\mathrm{e}}$ scan, (runs $g-l$ )

$d 3 d 10 n-2.45 \mathrm{GHz}$, NQS batch for Power scan, (runs $i-k$ )

$d 3 d 11 n-2.45 \mathrm{GHz}$, Variations of $d 3 d 109 n$ after the workshop. 


\title{
Appendix F
}

\section{Memo on FWP Issues}

February 26, 1993

\author{
memorandum
}

To: Distribution

From: $\quad$ M. E. Fenstermacher

Subject: $\quad$ Tasks to be done before FWP Presentations on LHCD in DIII-D.

Several physics questions continue to be raised about the application of the 2.45 GHz LHCD system from ASDEX on DIII-D. This memo will describe what we will try to do to get some answers to these questions before the Field Work Proposal presentations at the end of next month. Given the short time, there will still be outstanding questions at the time of the presentations and our FWP will describe plans to answer those by the end of FY94, before large expenditures are made towards DIII-D installation. However, the following may be addressable in the short term:

1. It has been pointed out that all of the ray-tracing modeling work so far has been done for rays that began very near the separatrix. In the real experiments, the antenna will be resessed somewhat into the SOL plasma. The concern is that we have not verified that the power will indeed couple well at the antenna and that the LH wave "cone" will not propagate to the separatrix.

Task 1a.: A search will be made to see if modeling of the SOL has been done for the VH-mode plasma used in the LHCD analysis (Shot 75121). This will provide characterization of the SOL plasma from which it can be determined if the LH wave propagation conditions are satisfied.

Task 1b: Previous experience on $\mathrm{LH}$ antenna coupling will be checked to determine the optimum density in front of the grill mouth to minimize reflectivity for the conditions ( $\mathrm{N}_{\|}$spectrum, frequency) proposed for the DIII-D experiments. This, combined with 1a above, will allow us to specify where the antenna would have to be located for the Shot 75121 (VH-mode) plasma. 
2. It has been suggested that the "density limit" observed in many LH experiments is not really a limit on the absolute value of the density but is instead a limit on the density gradient. If this were the case, the large gradients in DIII-D VH- and $\mathrm{H}$ - modes might produce degradation of the $\mathrm{LH}$ waves even for low densities.

Task 2: The way to clear up this issue is to find data from experiments with $\mathrm{LH}$ injected into a good H-mode plasma. The available database from JET , JT-60, JT60U, and ASDEX will be checked. We will search for H-mode experiments with large density gradients measured near the plasma edge in which the density was below the conventional $\mathrm{LH}$ limit given by $\mathrm{n}_{\mathrm{e}}\left(10^{19} \mathrm{~m}^{-3}\right)<\mathrm{f}(\mathrm{GHz})^{1.6}$.

3. The point continues to be raised that even if the "density limit" is explainable by parametric decay instability (PDI) theory, the theory is highly non-linear and predictions from calculations for the new, high dielectric regime $\left(\omega_{p e}^{2} / \omega_{c e}^{2}>>1\right)$ characteristic of counter current drive at the edge of a DIII-D Vh-mode plasma will need to be verified by experiment.

Task 3: This point was acknowledged at the informal workshop held at GA, $2 / 11 / 93$. To make some progress on this issue, the database of existing LH experiments in high density, low field conditions will be rechecked. We will document the observations made at MIT on VERSATOR-II at $2.45 \mathrm{GHz}$ and look again for DII-A LH data at $800 \mathrm{MHz}$. The goal will be to find data at high enough density to have the dielectric substantially above one, while at the same time satisfying the LHCD and accessibility conditions, $\omega>2 \omega_{L H}$ and $N_{\|}>$

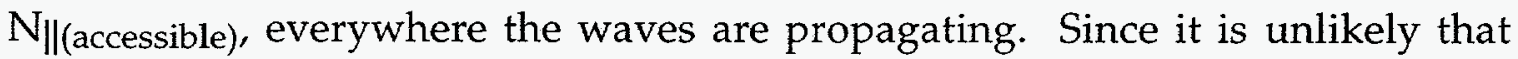
much data of this type exists, we will continue to explore the possibility of doing near term experiments on JT-60U in this regime by lowering the toroidal field. 


\section{Appendix G LHCD Section of LLNL FWP}

\section{Proposal for a Lower Hybrid Current Drive System}

We propose here to install a $2.45 \mathrm{GHz}$, Lower Hybrid Current Drive (LHCD) system on the DIII-D tokamak to enhance the existing capability to modify and control the current density profile. We intend to optimize the LHCD system to drive current in the outer regions of the plasma thereby complementing the existing capabilities of the Electron Cyclotron Harmonic (ECH) and Fast Wave (FW) current drive systems for driving current in the core of the plasma.

The Advanced Tokamak (AT) program on DIII-D focuses on four modes of plasma operation which have produced either enhanced confinement and/or high beta stability under transient conditions. Known as the VH-mode, the high $\mathrm{l}_{\mathrm{i}} \mathrm{H}$-mode, the second stable core mode, and the high ebp mode, each is thought to have a distinct current density profile. To extend these improved performance modes to long pulse and steady state operation requires that techniques be developed to modify and control the current profile with non-inductive current drive systems.

The existing current drive program on DIII-D includes Neutral Beam (NB) injection, Electron Cyclotron (EC) harmonic waves, and Fast Waves (FW) in the ion cyclotron frequency range (ICRF). The combination of these systems allows the DIII-D program to modify the current density profile in the plasma core (near the plasma axis and out to about $2 / 3$ of the plasma radius). However, none of these systems has shown a high enough current drive efficiency to affect the current density profile near the plasma edge where the temperature is comparatively low.

Control of the edge current density profile is thought to be critical to extension of several of the AT modes to longer pulse length (in particular the VH-mode and the high $l_{i} \mathrm{H}$-mode). The comparatively high efficiency of Lower Hybrid (LH) current drive lead to initial studies of the feasibility of modification of the current density profile in this region on DIII-D with a LHCD system.

In addition to comparatively high efficiency, as shown in many previous experiments on various tokamaks, there are several other advantages to the use of LHCD for edge current density modification over other systems. The damping of the LH wave spectrum required to drive current in this region is very strong so that the wave power and resulting current can be localized. The wave damping and resulting driven current has been well simulated by computer codes in previous experiments, so that an optimized system design can be developed for the particular LHCD experiments planned on DIII-D. Finally, the power sources and other components of the necessary LHCD system for the DIII-D application are readily available and have been proven on previous experiments at ASDEX and PLT. 
Our approach will be to perform a detailed engineering design and costing evaluation of the use of the ASDEX $2.45 \mathrm{GHz}$ system on DIII-D by the end of FY 94. This will incorporate results of physics analysis of the optimum LHCD system parameters for the modification of the edge current density profile in present and future planned DIII-D VH-mode and high $\mathrm{l}_{\mathrm{i}} \mathrm{H}$-mode plasmas. Modification of the system for the DIII-D application will follow in FY 1995 with installation planned for FY 1996.

Our initial physics feasibility studies of LHCD in DIII-D were presented in the report for milestone \#18 of the present LLNL/GA collaboration, UCRL-ID111838, September 1992. Subsequent refinements of that analysis were reported at the US/Japan CD Workshop held at PPPL, December 14-16, 1992, and at an Informal Workshop on LHCD at GA, 2/11/93 (A report summarizing the findings of this workshop is in progress). Calculations using several different codes agree that use of the $3 \mathrm{MW}, 2.45 \mathrm{GHz}$ ASDEX LHCD system on DIII-D would allow noticeable modification of the edge current density profile for $\mathrm{VH}$ mode conditions. There is however, little margin between the predictions and the requirements for substantive change in the current density profile, leading to the conclusion that additional power would likely be needed to assure control over the edge profile. Also, the $1 \mathrm{sec}$ pulse length of the present system was thought to be adequate for initial experiments, but a near term goal of 2-5 sec operation and eventual 10 sec operation was highly desirable.

The preliminary engineering evaluation of the necessary modifications to use the ASDEX system on DIII-D were discussed at an informal workshop at GA, $10 / 20 / 92$, and also at the recent workshop, 2/11/93. For all edge current drive applications, some form of new launcher would be required. In addition, 2 of the $60.5 \mathrm{MW}$ klystrons are limited to $1 \mathrm{sec}$ operation at present; and would likely require reconditioning to run for long pulses. Other components of the system such as the circulators and elements of the waveguide splitting network would require upgrading to extend the pulse beyond the current $1 \mathrm{sec}$ limit used on ASDEX. Some of the necessary long pulse hardware may be available at PPPL.

For the remaining part of FY 1993, we plan to refine the physics evaluation including comparisons of single and double null plasma operation with LHCD, optimization of the required $n||$ spectrum and choice of the optimized launcher port location. These studies will be done using plasma profile data from present and planned $\mathrm{VH}$-mode and high $\mathrm{l}_{\mathrm{i}} \mathrm{H}$-mode experiments. Engineering evaluation of the necessary upgrades to extend the ASDEX system to greater that 1 sec pulse length will be completed. Refinements of the cost estimates for system modifications, upgrades and installation will also be done.

A detailed engineering evaluation and costing for the modification and installation of the ASDEX system on DIII-D will be done by the end of FY 1994. Detailed physics scenarios and experimental plans will also be developed for VH-mode and high $\mathrm{l}_{\mathrm{i}} \mathrm{H}$-mode experiments with LHCD during this time. 


\section{Appendix $\mathrm{H} \quad$ Viewgraphs on Frequency Choice and Parametric Decay Instabilities}




\section{LOWER HYBRID CURREMT DRIVE THEORY}

Considerations of Accessibility, Density Limit, Frequency Choice and Parametric Decay in the DIII-D LHCD Proposal

M. Porkolab

$$
\begin{aligned}
& \text { ACCESSIBILITY } \left.\quad \text { (DETERMINES } N_{\| 1}\right) \\
& \frac{\omega_{\mathrm{pl}}}{\omega}=M_{11} Y-\sqrt{1+M_{\| 1}^{2}\left(Y^{2}-1\right)} ; \quad Y=\frac{\omega}{\sqrt{\omega_{\mathrm{ce}} \omega_{\mathrm{cl}}}}
\end{aligned}
$$

OR

$$
N_{|| 1}=\frac{\omega_{p 1}}{\omega} Y+\sqrt{1+\frac{\omega_{\rho 1}^{2}}{\omega^{2}}\left(Y^{2}-1\right)}
$$

OR

$$
N_{\text {ll }}=\frac{\dot{\omega}_{\mathrm{pe}}}{\omega_{\mathrm{ce}}}+\sqrt{1+\frac{\omega_{\mathrm{pe}}^{2}}{\omega_{\mathrm{ce}}^{2}}\left(1-\frac{\left.\omega_{\mathrm{ce}} \omega_{\mathrm{cl}}\right)}{\omega^{2}}\right.}
$$

\section{LAMDAU DAMPING (DETERMINES $N_{1 / 2}$ )}

$$
\begin{array}{ll}
\text { QUASI-LINEAR: } & N_{1 / 2} \simeq 7 / \sqrt{T_{\mathrm{e}}(\mathrm{keV})} \\
\text { LINEAR: } & N_{1 / 2} \simeq 5.4 / \sqrt{T_{\mathrm{e}}(\mathrm{keV})}
\end{array}
$$

Presented at the LHCD Workshop

General Atomics, San Diego

February 11, 1993 


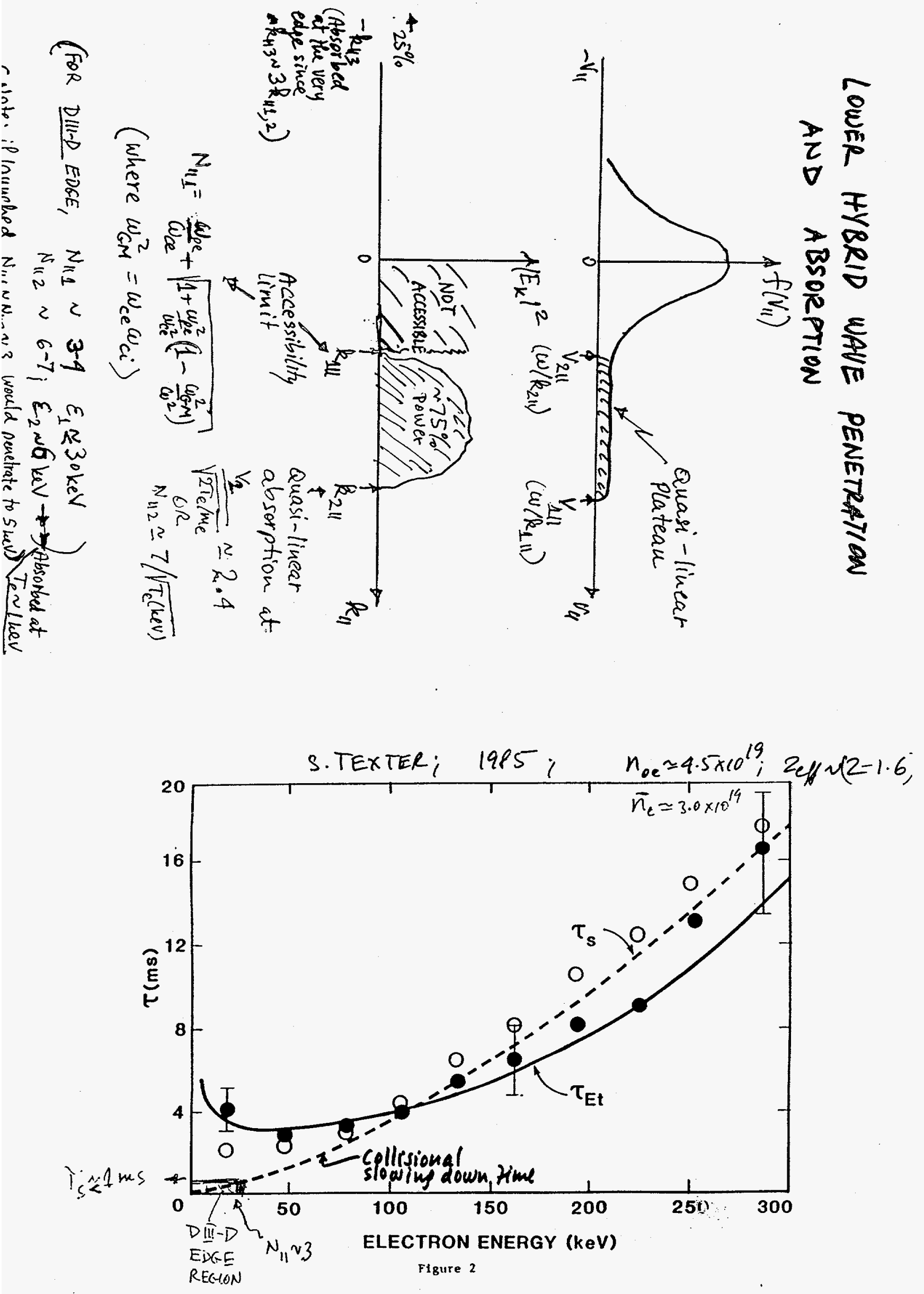



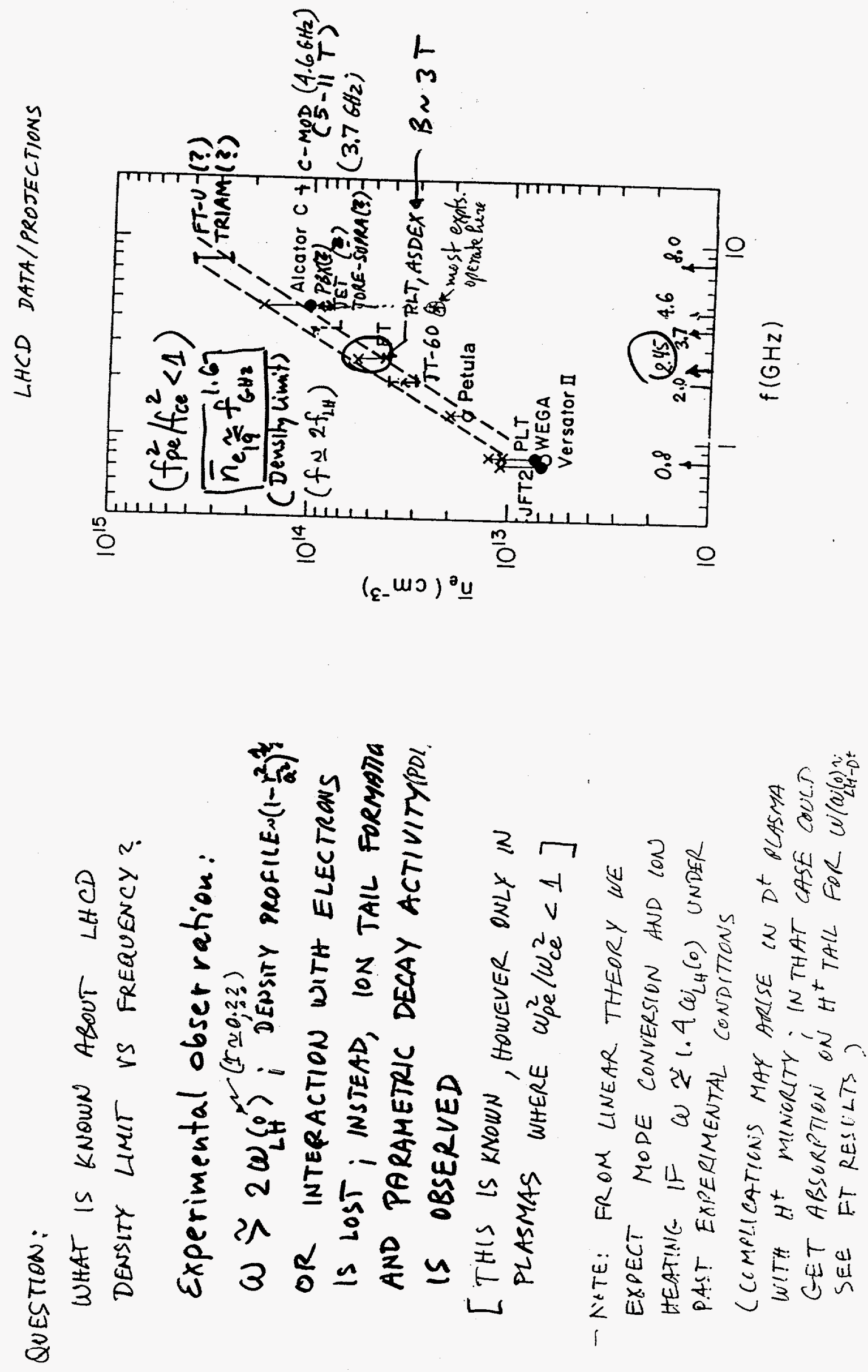


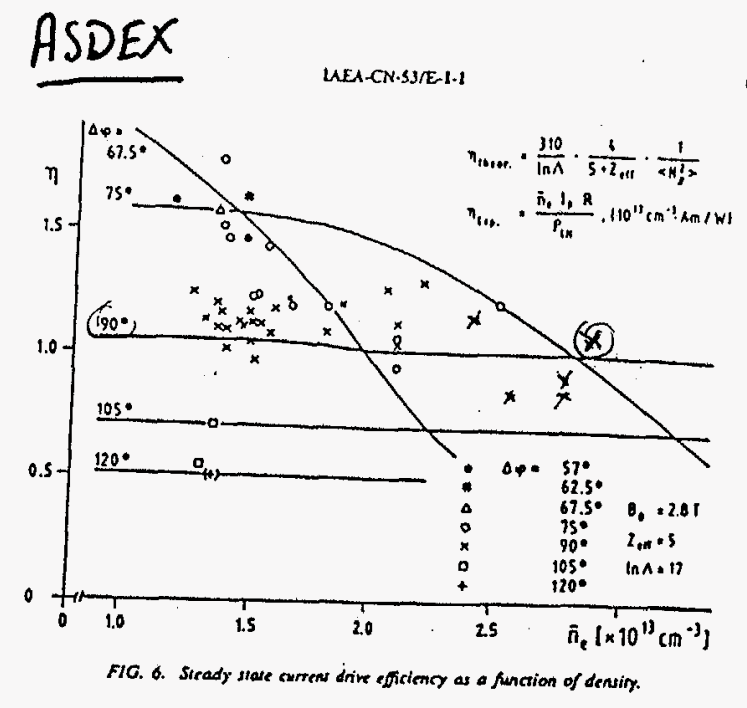

LEA-CN-SOVE-1.4

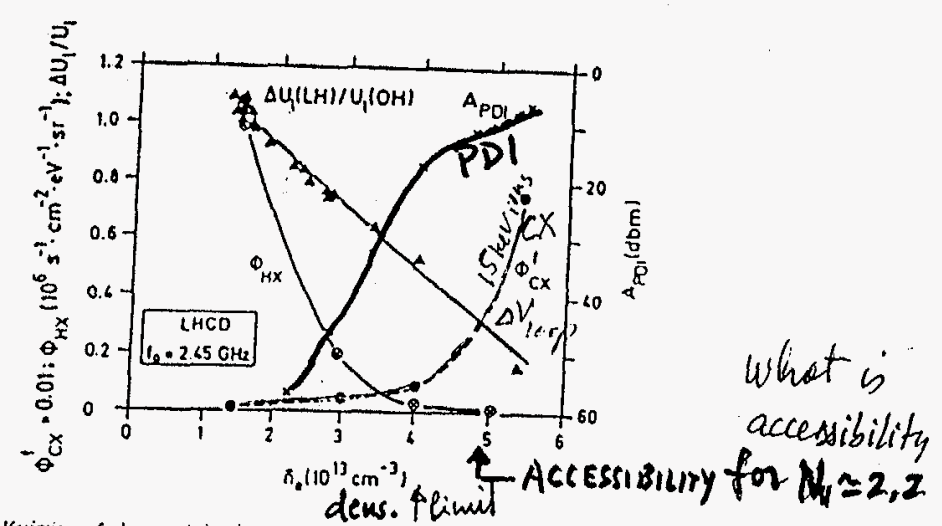

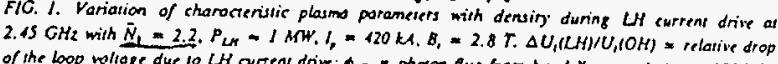


sautlitie in paramestic decay spectrem.

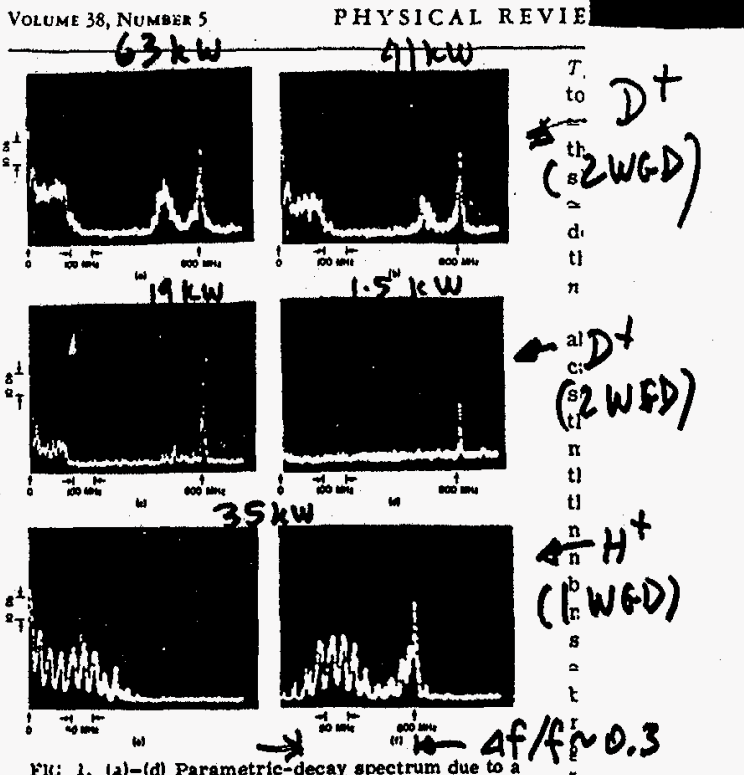

Fils 1. (a)-(d) Parametric-decay spectrum due to spitt -..Ave gulde; $D_{2} \mathrm{gas} ; \bar{\pi} \times 1.8 \times 10^{13} \mathrm{~cm}^{.3}$. (a) $P_{1 \text { in }}$ $263 \mathrm{~kW}$.

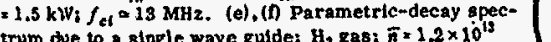
$\mathrm{cm}^{-3}, F_{i n}=35 \mathrm{~kW}$. (e) Low-frequency spectrum, and $]$ (f) pump and sideband: $f_{t} \approx 25$ MHO

The two sections of the split wave guide were driven in phase oppos ition (1.e., $180^{\circ}$ out of phase). The cross-sectional area of both wave-guide sys tems was $10 \mathrm{~cm} \times 20 \mathrm{~cm}$. The detalls of the heating results were reported recently. The movable probe was a coaxial shielded T-s haped electrostatic $r$ probe, with a lrequency response good up to $1 \mathrm{GHz}$ (hat within $3 \mathrm{~dB}$ ). The probe was located radially in the shadow of the limiter, but near the end of the expertments it was pushed several cont or the experinge This resulted in an $10 \mathrm{~dB}$ indcating that the waves originate irom points deeper in the plas than the normal probe position. During these experiments the plasma parameters were as follows: $B=15-20 \mathrm{kG}$ minor radius $a=17 \mathrm{~cm}$ and average density (measured by a microwave inter ferometer) $\pi_{0} \sim 5 \times 10^{12}-3 \times 10^{13}$ in $\mathrm{H}^{\prime}$ or $\mathrm{D}^{\prime}$ plas ma: The electron temperatures were typicalty Mr. PORKOLAB et al,
PRL $1 / 31 / 1977$
ATC, $0.80642 \cdot 1976$

volume 38. Numasas PHYSICAL
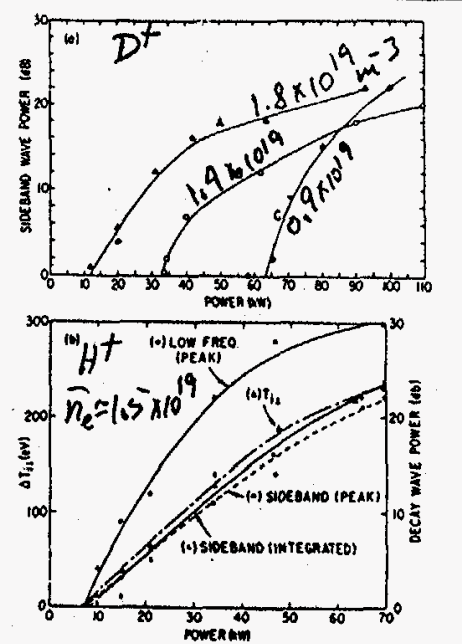

Fio. 2. (a) Stdebead emplitude vs $P_{\text {in }}$ for 2 splitwave-gulde coupler; $D_{2}$ gas. Curve $A, \bar{n}=1.3 \times 10^{13}$

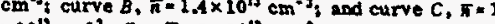
$\times 10^{14} \mathrm{~cm}^{-3}$. For $\pi<9 \times 10^{12} \mathrm{~cm}^{-3}$ and $P<100 \mathrm{~kW}$, Do decay is observed. (b) $\Delta T_{11_{2}}$ and decay-wave emplitud

$P \$ 120 \mathrm{~kW}$. In $\mathrm{H}^{*}$ plasma no decay was observ for $A_{0}<6 \times 10^{12} \mathrm{~cm}^{-3}$ or $\omega_{0} / \omega_{u m}^{\max } 21.40$. Of course, if decay occurred radizlly out ward tro! the center, locally higher values of $\left\{\omega_{\mathrm{g}} / \omega_{\text {Lx }}(r)\right\}$ may be allowed. However, the fact that there i a rather sharp low-density limit beyond which parametric decay was observed, shows that de perimental observation suggests that parametri decay (at least for the main part of the spectru) must occur within the bulk of the plasma, l.e., mostly near densities $n>6 \times 10^{12}$ (but not necess nly at the center). Thus, we must be observing If sigrals on our probe which propagate out of 1 interior. We also remark that the very-low-irt quency part of the spectrum (1.e., $\omega<\omega_{\text {eis }}$ ) had sometimes a lower densily threshold than the higher-frequency components; thus, this part o radially possibly due to the portion outside er coupled to the fast the portion of pump po densities this part of the spectrum was typically 20-30 dB down from the pump-wave amplitude 



FIG. 3. Frequeocy (w) add growth rate $\left(\gamma_{0}\right)$ as a func-

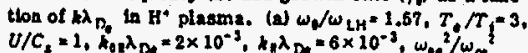

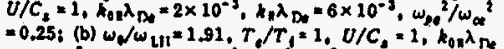

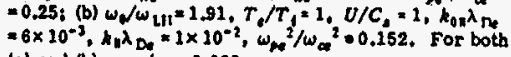
(a) $\operatorname{cod}$ (b), $\omega_{c 1} / \omega_{1}=0.033$

point of the sideband waves $\omega_{2}$ (i.e., the transition point (rom backward to forward waves). In general, the numertcally obtalned decay spectra

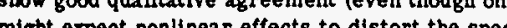
might An important theoretical question is the effect of nonuniformities of the pump wave upon the inwave is confined by resonassume that the pum wave is corted by the wave cones to a finite stabilization elimates wost instabilitins for $\omega_{M H}>2 . \omega$ On the contrary, for $\omega_{0} / \omega_{L H}<2$ decar into the hot fon plasma waves (the short-wave length forward-wave branch in Fig. 3) can occur due to the low convective losses of these waves. In addition, decay into the backward-wave branch may a lso occur if the sidebands form standing modes in the toroidal direction near the rational magnetic surfaces (these modes have recently been discussed by Coppi et al. $\left.{ }^{\natural}\right)$. Again, the condition for the existence of these modes is that $\omega_{0} /$ $\omega_{\text {LIt }} \approx 2$. In thls case, convection due to shear de that the threshold due to shear is $U / C, \approx 0.5$. The foregoing considerations for the ATC parameters predict threshold power in the range of $P_{1 \mathrm{n}} \approx 10$ experimental observations.

Our analysis also indicates that whereas for $T_{d}$ $T_{i}=1, \operatorname{Im}_{X_{i}}(\omega) \sim \operatorname{Im}_{\chi_{e}}(\omega)$, we have for $T_{e} / T_{i}=3$, ity of species $j$ l. Since according to quas ilinear theory, heating of a particular species of partimatn body of lons are heated by the low-frequen. cy ion-cyclotron quasimodes (i.e., where $T \approx \tau$ ) On the other hand, we expect that the sideband heats mainly the parallel component of the elec trons, and the perpendicular tall of the ions. For $T, \angle 3 T_{\text {th }}$ even the low-frequency quasimodes bined with the experimentally measured density and temperature profiles, these results suggest that in ATC parametric decay and ion heating should occur in the region $0.5 \leqslant r / a \leqslant 0.8$, where $2 \approx T_{0} / T_{1} \approx 1$, and $n(r) \propto \frac{1}{2} n_{0}{ }^{\text {nas }}$. Ot course, strong turbulence effects may may modify some of these conclusions; for example, the sideband may also Interact with the main body ion temperature the wave amplitudes are sufficiently large: $A$ more ietall $f$ account of this work will be reported elsewher.

One of us iM.P.) wishes to thank the Alexander von Humbols. Foundation for an honorary fellow ship at the $N$ ax-Planck-Institut, Garching, Gercarried aur or the theoretical work was cing rating crew of ATC.

\section{(a)}

-Work supported by the U. S. Energy Research and


3073.

T. H. Sttx, Phys. Rev. Lett. 15 . 878 (1965): V. E. Golant, Zh. Tekh. Fiz. 41,24

${ }^{2}$ K. Bol et al., Phys, Rev. Lett. 29, 1495 (1972). 'S. Bernabel et al.. in Proceedings of the Thlrd Sym (to be published).

M. Porkolab, in Proceediogs of the Third Symposium on Plasma Heating in Toroidal Devices, laternational School of Plasma Physics, Vareana, italy, 1976 (to be published).

porkolab, Phys. Flutds 17, 1432 (1974), and also in Proceedings of the Sympostum on Plasma Heating in 1974), pp. 28-49.

B. Coppi et al.. Nucl. Fusion,16. 309 (1976). $I_{m} x_{\theta}(\omega) \rightarrow \operatorname{Im} x_{i}(\omega)$ [ where $x_{,}(\omega)$ is the susceptibil should heat the bulk electron temperature. Composium on Plasma Heating in Toroldal Devices, Inter-
AlcaTor - $C ; 9.66 \mathrm{H}_{2}$;

exist since if power has to spread over a larger surface area. The experimental situation should be somewhere betwoen these two extremes. Near the waveguide mouth we expect stroag resonance cone pattern; however, as the wave propa gates farther into the plasma the distortion of the resonance cones bocomes progressively more severe, and eventually the resonance cones should be completely destroyed after several toroidal transits. This picture is supported by $\mathrm{CO}_{2}$ lase scattering data, at least at relatively high densities where parametric docay is observed. A quantitative prediction of he threshold for his case is rather diche fllow the decay wave along its rajectory unil enough

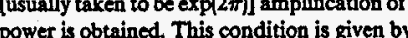

$$
\int_{0}^{5} \frac{r(5)}{\left|v_{2 b}\right|} d \zeta=\pi
$$

where $\zeta$ is the coordinate along the trajectory of the decay wave and $u_{2}$ is the component of the decay wave group velocity along the trajoctory. In addition to changing plasm parameters, along the ray trajectory the pump electric field is varying because of resonance cone spreading. A better estimate could be made with the aid of a ray tracing code the includes the effects of seattering by density fuctuations. " Below, we shall give some representative examples of the threshold if power by assuming a homogenous plasma bet different spatial distributions of the pump wave. In reality, the decay wave will not stay at the radial location where the growth rate is maximum, but will propagate to regions of different plasma parameters and lower growth rate, which contributes to raising the ald above the value calcula

First, we consider the case with undistor,ed resonance cones. There is an optimum angle of propagation $\theta$ that $k_{1}^{-}$ makes with $k_{01}$. This is determined by the competitio reducing the convective loss. We shall use the coupling constant given by $\mu^{-}$in Eq. (5) of Ref. 11, namely,

$$
\mu=\frac{e}{m_{e}} \frac{k}{k}\left[\left(\frac{k_{1}-E_{01}^{2}}{\omega_{0}^{2}}\right)^{2}+\left(\frac{k_{1}-E_{01} \sin \theta}{\omega_{00} \omega_{c o}}\right)^{2}\right]^{1 / 2} .
$$

Two important contributions to the parametric coupling constant are that caused by the $E \times B$ motion of the electrons (the second term, which varies as $\sin \theta$ ) and that caused by the parallel motion of the eloctrons driven by $E_{1}$ (the first erm, which is independent of $\theta$ and dominates for small angles,' $\left.\sin \theta<\omega_{p e}^{2} / \omega_{d} \omega_{\infty s}\right)$. The polarization drift term (which varies as $\omega_{3} \theta$ ) is salles than the paralled term by at least a factor $\omega_{p}^{2} / \omega_{\text {e }}^{2}$. Near the plasma edge we find that hecon Thecouling for his ase is caused by $E$. This is


$\simeq a$ where $X \times 1$ coupling dominates over the $E_{1}$ coupling in a hoce $x$ tive parameters of deuterium plasma, $B=62$ T

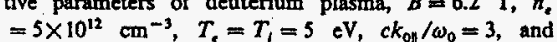
$=5 \times 10^{12} \mathrm{~cm}^{-3}, T_{c}=T_{i}=5 \mathrm{eV}, \quad c k_{00} / \omega_{0}=3$, and guide mouth in Alcator $C$ where the $E_{q}$ coupling term is
PAAXS. FLUIDS, 28 , Takase, Porkolas, Schuss ot al


is $P_{\mathrm{r}} \simeq 50 \mathrm{~kW}$. However, we note that the distance requirod for the decay wave power to amplify by exp (2m) is approximately $4 \mathrm{~m}$, which is roughly one complete toroidal revolution. Account must also be taken of the decreasing $E_{1}$ driven growth rate as the wave propagates in to the plasma (see Fig. ). This will further raise the threshold. In order for the de

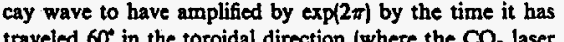
traveled 60 in the toroidal direction (where the $\mathrm{CO}_{2}$ laser scaltering data were oblaincel, a growth rate of $\gamma$ f nearly $2 \mathrm{MW}$.

In the second limit, if we assume that the pump wave spreads over the entire fux sufoce uniformly, the pump wave electric feeld is reduced by the pump $\left[\left(2 \pi^{2} R a^{2}\right) /\left(2 L, L_{x}\right)\right]^{1 / 2}$ from the case of resonance cone propagation. Here $2 \pi^{2} R a^{2}$ is the surface area of the flux surface at $r=a$, and $2 L, L$, is the cross-sectional are of the two resonance cones (where $L$, and $L$, are the dimensions of the waveguide array in the poloidal and toroidal directions, respectively). We shall take representative edge parameters of deuterium plasma, $B=6.4 \mathrm{~T}, n_{t}=0.9 \times 10^{14} \mathrm{~cm}^{-3}, T_{c}=T_{t}$ $=20 \mathrm{cV}, c k_{01} / \omega_{0}=3$, and $c k-/ \omega_{0}=7$, corresponding to the peak of the growth rate in Fig. 5 . The homogeneous plas. ma threshold under these conditions is $P_{\mathrm{rr}} \approx 1 \mathrm{~kW}$. The threshold $\mathrm{rf}$ power for this case is $2 \mathrm{MW}$. This model is not cupported by the $\mathrm{CO}_{2}$ laser seatlering data. At 60 away toroidally from the wave launcher during its first pass, the pump wave appears to be still fairly well localizod ${ }^{18}$

\section{RADIO FREQUENCY PROBE MEASUREMENTS}

The externally launched lower-hybrid pump wave, as well as parametrically excited lower- and upper-sideband lower-hybrid waves and low-frequency quasimodes, were
Setup

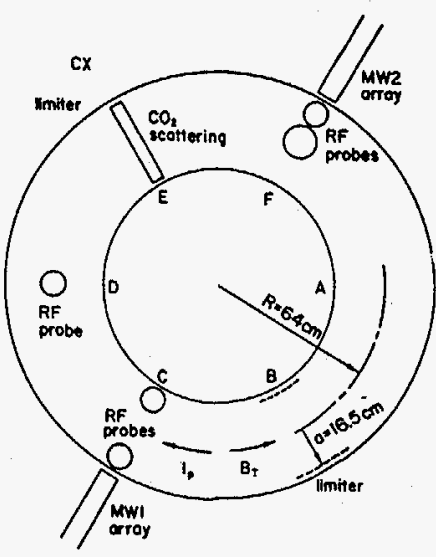

FIG. 7. Locations of the waveguide arrays, limiters, and reterant tiagnos- 


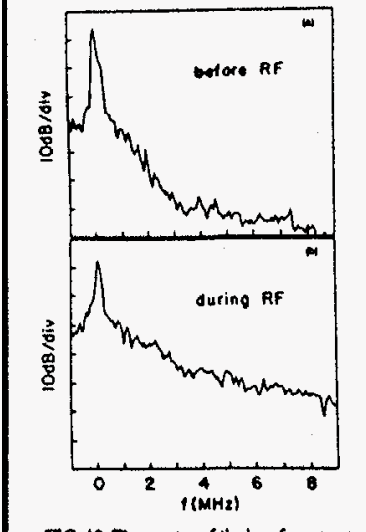

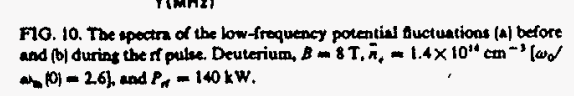

We show the frequency width (FWHM) of the broadened pump wave as a function of density for deuterium and hydrogen plasmas in Figs. $9(a)$ and $9(b)$. The frequency width is indepeadent of the magnetic field, and is also fairly independent of the injected if power level. There is also a frequency down thift of the order of $1 \mathrm{MHz}$ except at low densi-
ties, $n_{0} 5.1 \times 10^{14} \mathrm{~cm}^{-3}$, where the spectrum is symmmetric about the pump frequency. In Figs. 10(a) and 10(b) we show the spoctra of the low-frequency potential fuctuations be fore and during the of pulse. There is significant increase in the Auctuation level in the range of a few MHz. There is a threshold density $n_{n} \approx 1 \times 10^{14} \mathrm{~cm}^{-1}$ below which this enhancement in the potential fluctuations is absent. There is also a power threshold of 1 few tens of $k W$ for this enhance ment. These results suggest that parametric decay into ionsound quasimes" may be oceurring. We note that neither the ion-density ductuation observed on ion saturacion cur

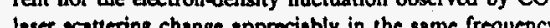
laser satering change appreciably in the same trequency not in conotradietion with parametric exciution of ion an quacimodes "but exclude the possibility that this potential avcausion enhancement is entirely caused by enhancement in drift wave turbulence. The frequency broadening of the pump is ausod by seattering from low-frocuency density tuctuations ${ }^{13}$ at densities below threshold. It is not clear a present how much broadening, if any, is caused by parame. tric decay at densities above threshold.

\section{B. lon-eyctotron eldebands}

The high-frequency and low-frequency spectra over a wider frequency range at two different densities, one above The ion-cyclotron peaks are separated from each other by

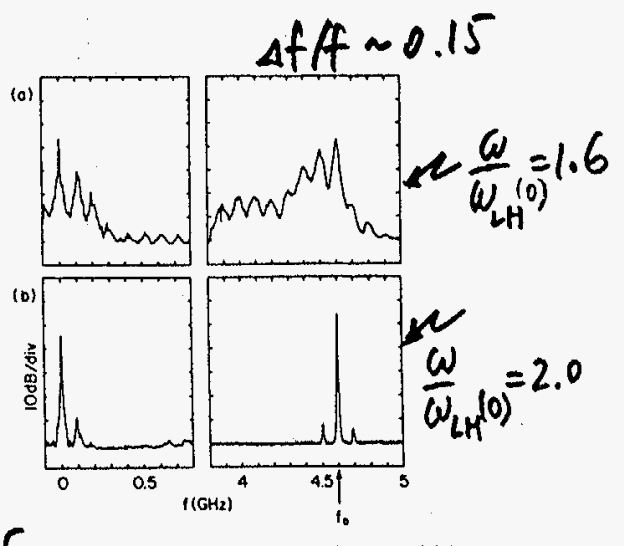

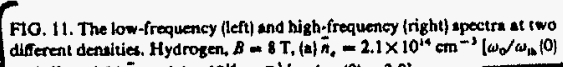

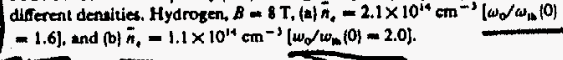
outside (larger major radius side) edge of the torus, suggestoutside (larger sajor hands were generated near the outside ing than these ing betwoen ion-cyclotron peaks is independent of the probe location along the major radius in the range $52<R<76 \mathrm{~cm}$ (the major radius of the torus $R_{0}$ is $64 \mathrm{~cm}$ ). There are two distinct features in the high-frequency spectrum of Fig. 11(a). Based on our numerical results presented in the last section, we interpret the first few harmonic lower-sidebands to be caused by parametric decay into ion-cyclotion quasimodes $\left(\omega \simeq n \omega_{c}\right)$ near the plasma edge where the temperature is low, and the higher harmonics, peaked around $\left|\omega^{-}\right|$ $\omega_{0} \approx 0.85, t o$ be caused by parametric decay into nonresonant quasimodes $\left[\omega / k_{1} v_{k s} \simeq O(1)\right]$ excited farther inside where the temperature is higher. ${ }^{\circ}$ The higher harmonics have a higher density threshold than the lower harmonics. In contrast to the lower-sideband spectrum, the low-frequency spectrum is always monotonically decreasing and never shows a peak at $\omega / \omega_{0} \simeq 0.15$. In Aleator $C$, the douvinan peak is almost always the first ion-cyclotron lower-sideband.

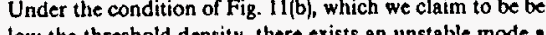
low the threshold densily. there wists an ung $\omega=\omega_{r}$ again, $\omega$. plamp. The is metric lower- and upper-sidebands are observed. Webelieve that rather than being caused by parametric decay, thesc sidebands are produced by the passive nonlinear beating of the lower-hybrid wave on a pre-existing marginally unstable ion-cyclotron wave. The amplitudes of these modes ate small ( $50 \mathrm{~dB}$ down from the pump) in contrast to the parame tric modes in Fig. 11(a). If these ion-cyclotron modes were only marginally stable in the density regime where we observe parametric decay, there is a possiblity that they might be driven unstable in the presence of the pump wave, thus reducing the threshold for parametric decay.
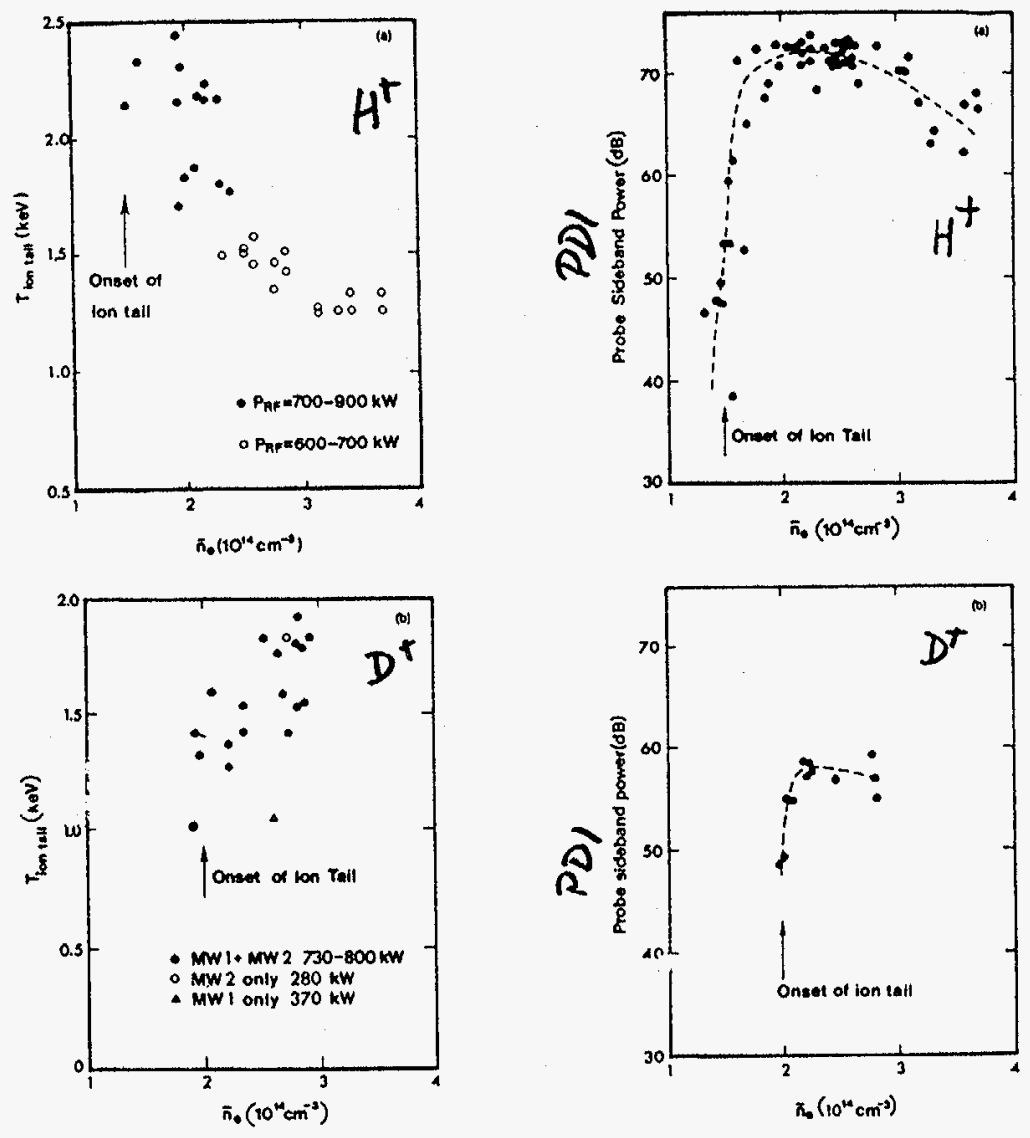

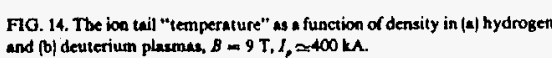

The parametric threshold density increases with plasma current. This scaling with current has been previously ion tail threshold coincides very well with the parametric threshold we have recently observed a formation of an ion tail below the parametric threshold density in a hydrogen plasma at $B=10 \mathrm{~T}$ and $l_{e}>450 \mathrm{kA}$. At these high currents direct absorption of the pump wave by hydrogen ions further inside the plasma may be taking place. Detailed studies of this high current regime and the role of minority hydrogen absorption in deuterium plasmas will be underalen in the near future and the results will be presented in a later publ-

(n)




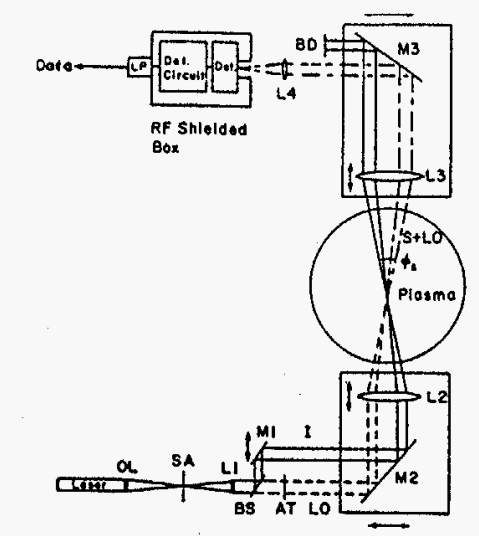

FiG. 16. The $\mathrm{CO}_{2}$ vattertas configuration.

needed for heterodyne derection, ${ }^{24}$ passed vertically through the plasma at a distance $x=2 R-R_{0}$ from the plasma center along the major radius, and was beld fixed during a $k$ scan to ensure constant detection efficietcy. The main laser beam was oriented parallel to the $L O$ beam at mirror $M 1$, and the wes located inside the plasme volume. The main laser beam scattered in the direction of the LO beam, was detected and optically mired with the LO beam at the Hetector (Ge:Cu photomixer). By translating the mirror $M 1$ vertically, the separation between the two beams could be altered. The spacing between the two beams $d$, and the focal length $f$ of the lens $\mathrm{L} 2$ determines the scattering angle $\phi_{1} \approx d / f$, and therefore the wavenumber of the wave being studied, $k \simeq k_{1} \phi_{1}$, where $k_{1}$ is the wavenumber of the $\mathrm{CO}_{2}$ laser beam. We note that in the present configuration only waves with wave vectork oriented along the major radius could be deteetod. The typical range of wavenumbers studied were in
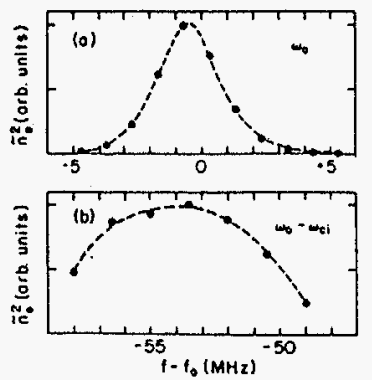


$-280 \mathrm{~kW}, x=+12 \operatorname{com}, \operatorname{ard} k=180 \mathrm{~cm}^{-1}$
-1

001 Ptys. Furos, Vol, 28, No. 3, Maren 1985

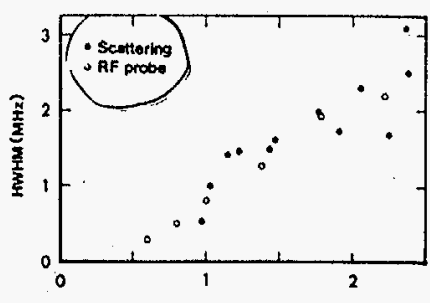

$\bar{n}_{0}\left(10^{4} \mathrm{~cm} / \mathrm{cm}^{2}\right)$

FG. 18. The density dependence of the frequency width (HWHMM) of the probec The probe was loceaied $180 \sigma^{2}$ away toroidally from the $\mathrm{cm}^{-1}$ ) and $n$ so that it is insensitive to the surfice waves. Deuterium, $8<B<10 \mathrm{~T}$.

the range $80<k<240 \mathrm{~cm}^{-1}$. The waist radius of the laser beam at the focus was $0.1 \mathrm{~cm}$, which gives a horizontal spatial resolution of $\pm 0.1 \mathrm{~cm}$ and a wavenumber resolution of $\pm 20 \mathrm{~cm}^{-1}$. The vertical spatial resolution varies inversely $k=200 \mathrm{~cm}^{-1}$ it wousured to be $\pm 10 \mathrm{~cm}$ at $k=80 \mathrm{~cm}^{-1}$ (at $k=200 \mathrm{~cm}-1$ would be $\pm 4 \mathrm{~cm}$. " The seatlered signal was analyzed using a 16-channel ilter bank for the pump

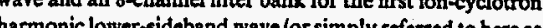

B. Scattering results

Typicial frequency spectra of the pump wave $\left(\omega_{0}\right)$ and the decay wave (first lont hes a ider frequency with the a

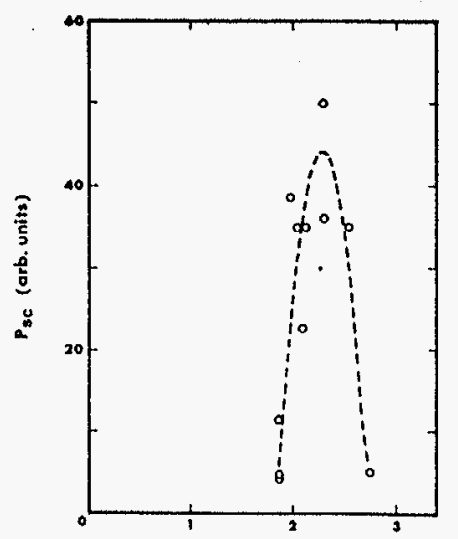

$\bar{n}_{0}\left(10^{14} \mathrm{~cm}^{-3}\right)$


$\mathrm{CO}_{2}$ scatterin
$k=140 \mathrm{~cm}^{-1}$

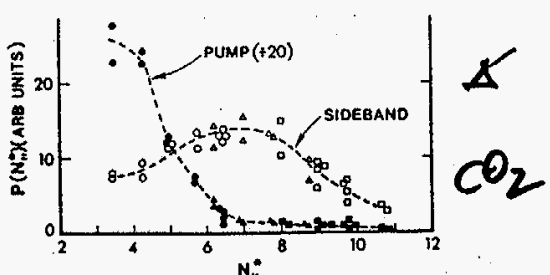

FO. 21. The power density spectre $P(N)$ for the pump wave and for the


The purmp wave deta points are scaled down by a iactor of 20 . Here $N_{i}$ defined in the tere.

numerical simulations) and $N_{f}$ is given in Ref. 18. In the present case this difference $\left(N_{1}-N\right.$ i $) / N_{1}$ should be smaller than for the case of Ref. 18 since, because of the larger values of $k$, the vertical resolution is better.

The power density spectra $P\left(N_{*}\right)$ for the pump wave and for the decay wave deduced from the experimentally measured $k$ spectra are shown in Fig. 21. These data were obtained al $x=+12 \mathrm{~cm} / x / a=+3 / 4$, and $10^{-1}$. arate $k$ scans covering the range sos $k$ sad in amo

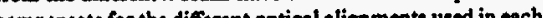
con an il hicher on an higher measured $k$ spectrum of the docay wave is consistent with

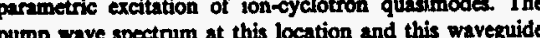
phating is peaked ot $N$ t 26 (which corresponds to the phaseing is peaked at $N_{i} \approx 2.6$ (which corresponds to the noted earlier, the difference between $N$ and the average value of $N$ (which is caused by uncertainty in the local plase ma density) is less for the present case since the vertical resolution is better at larger values of $k$. If we assume that the $k$, distributions of both the pump wave and the decay wave are isotropic in the perpendicular plane, the frequency- and wavenumber-integrated power in the decay wave is at most $3 \%$ that of the pur p wave. This would be an underestimation of the relative decay wave power compared to the pump wave power if $k_{r}>k_{\theta}$ for the pump wave and/or $k_{\theta}>k_{\text {, for }}$ the decay wave. Similarly, there could be significantly more power contained in ligher lon-cyclotron harmonic sidebands which were not studied by scattering in the presen experiments.

The wavenumber of the decay wave is characterized by $|k-| \leqslant 190 \mathrm{~cm}^{-1}$, which is consistent with the condition $\left|\omega-/ k_{1}-v_{*}\right| z 4$ at $x / a \simeq+3 / 4$, where $T_{c} \simeq 250 \mathrm{eV}$. The decay wave observod at $x / a=+3 / 4$ probably onginated near the waveguide mouth and propagated inside the pump "resonance conce" Therefore growth of the decay wave is infuenced by the growth rates at all locations between the wavegulde Tandan de

993 Ptys. Fludds, Vol. 28, No. 3, March 1885 larger minor radii where the electron temperature is lower, they are heavily Landau damped by the time they reach the observation point. Consequently, the wavenumber spectrum s determined by the electron temperature at the observation point. The observed wavenumber of the lower-sideband decay wave (as well as the pump wavenumber) is too small to create ion tails at this location since $\left|\omega-/ k_{1}-v_{n}\right| \approx 10$ if we lake $T_{1} \approx 250 \mathrm{eV}$ at $r=12 \mathrm{~cm}$. However, sccording to our theoretical predictions the low-frequency :con-cyclotron quasimodes are always strongly damped by ions. Therefore the by ion-cyclotron damping of the quasimodes.

\section{SUMMARY AND CONCLUSIONS}

Using of probes and $\mathrm{CO}_{2}$ laser scattering we bave ob ese $\omega_{0}, \omega_{\text {in }}(0) \leq 2$ in the Aleator C tokamak The pump wave data obtained with if probes sufficiently far from the of source are consistent with $\mathrm{CO}_{2}$ laser scattering data obtained in the plasma interior. However, there are some differences in the decay wave data obtained with the two techniques. Comparison of the probe frequency spectra with our numerical anaiysis suggests that parametric excitations of ioncyclotron quasimodes, nonresonant quasimodes, and possibly ion-sound quasimodes are taking place.

The decay region is located near the outside flarger ma. jor radius side) edge, $x / a z+3 / 4$, and localized toroidally

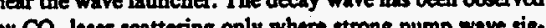
$\mathrm{CO}_{2}$ laver scalcengs by heavily ion-ayclotron damped quesimodes. The meat bearily ion-yclotron damped quesimodes. The mea$x / a=+3 / 4$ is peaked at higher values of $k$ (and hence $N$ ) than the pump wavespoctrumer the peak being determined by than the pump wavespectrum, the peak being determined by electron Landau damping at the lower sideband at the obserdeposited near the plasmas surface through excitation of par. ametric decay above the threshold density.

The experimentally observed threshold of power for parametric decay is an order of magnitude lower than the theoretically predicted convective threshold. The reason for this discrepancy is not clear at present.

The threshold density for parametric decay increases wellasma current. The onset of strong parame tric decay is well correlated with ion tail formation near the plasma edge, except at very high plasma currents $\left(I_{,}>430\right.$ kA) in hydrogen plasmas where the ion tail was observed below the parametric threshold density. These results and comparison with other experiments suggest that the density limit for electron interaction (current drive and/or electron heating) may be determined by more than one process. Thus, the operating conditions such as plasma current, toroidal field, density and temperature profiles, hydrogen or impuriin which mechanism will dominate.

Finally, we note that a conclusive verification of pump ey barametric decay instabilities was nor demonstatod here. Before more definite conclusions on 


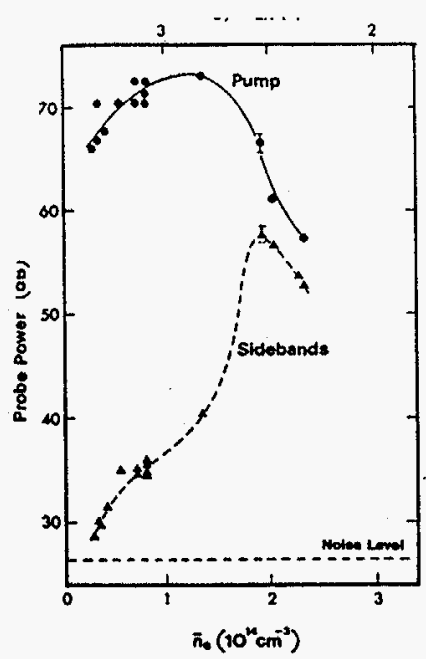

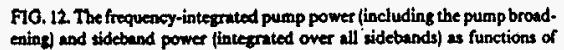

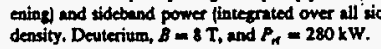

band power (integrated over all sidebands) as functions of density. Above the threahold denaily of $n_{0} \approx 15$ $\mathrm{cm}^{-3}$, the sideband power reaches about $30 \%$ of the pump power, and both sideband and pump powers decreasse rapid-
iy with density. In Fig. 13 we show the pump and sideband powers as functions of injected if power. Above the thresh

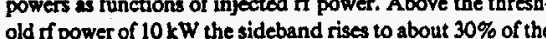
old $r$ power of $10 \mathrm{~W}$.hesideband rises to about $30 \%$ of the pump power, and both sideband and pump powers keep in
creasing linearly with rf power. Therefore we cannot concresde that pump depletion because of parametric decay has occurred. The decresse of the pump wave power at high densities may partially be caused by increased collisional damping near the plasma edge."

\section{Comparison with lon tail formation}

The correlation between ion tail formation and parame tric decay was studied with a mass-resolving charge exchange neutral analyzer located at the E port (see Fig. 7). The charge exchange $(C X)$ analyzer viewed the plasma pergap between a pair of larger major radius side through the $\mathrm{CO}_{2}$ laser scattering diagnostics was also located at this por The two waveguide arrays were located $60^{\circ}(M W 2)$ and $120^{\circ}$ (MWI) away toroidally from this port. At $B=9 \mathrm{~T}$ and $I$ $\simeq 400 \mathrm{kA}$, typical of eloctron heating regime, the ion taif were observed only above the sharp density threshold of $n$ $=-1.5 \times 10^{14} \mathrm{~cm}^{-3}$ in hydrogen [corresponding to $\omega_{d} \omega_{\mathrm{in}}(0)$ $=1.8$ and $\omega_{0}\left(\omega_{1 \mathrm{ta}}(a)=2.9 \mathrm{f}\right.$, and $n_{\mathrm{e}} \simeq 2.0 \times 10^{14} \mathrm{~cm}^{-3}$ in deuterium $\left[\omega_{d} / \omega_{1}(0)=2.2\right.$ and $\left.\omega_{0} / \omega_{1 k}(a)=3.6\right]$. This is

889 Phys. Fuids, Vol. 28, NO. 3. March 1985



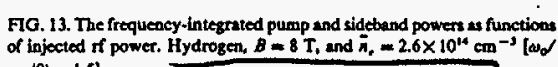
of injected ri power.
$\left.\alpha_{m}(0)=1.5\right]$.

ture" is plotted as a function of density. Here typical experimental values of $n_{c}(0) / \bar{n}_{e}=1.3$ and $n_{c}(a) / n_{s}(0)=0.3$ have been used to evaluate $a_{\mathrm{n}}$. Figures $14(\mathrm{a})$ and $14(\mathrm{~b})$ show the clear dependence of the ion tail threshold density on ion speces (H or D). The densily linh for the electron tail forma-

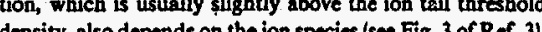
Thensity, also depends on the in species (see Fig. I of Rer. 3). in the bovecase is estored tobe ix abo casse is estinat be bess han $1 \%$ based on the CX nearrat meas is rents. Hydrogen ion tails observed in hydrogen pla mas sere lerram ion tills observed in derent plasmas. In general, stronger bals alls were observed when the lower-hybrid waves were injected from MW2 (the closer of the two arrays) rather than MWI, as can be seen by comparing the two open $\mathrm{CX}$ neutral fux, the flat ion tail profle obtained by $\mathrm{CX}$ updown scan, and the absence of neutron rate entancent the case of deuterium plasmas all imply that the ion tails did not originate from the plasma cenier. These density thresholds for ion tail formation correlated well with the onset of strong parametric decay observed by if probes. The frequency-integrated sideband powers observed by the rf probe during the same runs are shown in Figs. 15(a) and 15(b). These results suggest that ion tails are created near the plasma edge through the excitation of parametric decay, and that this effect is localized toroidally near the wave launching location. This toroidal localization is supported by the $\mathrm{CO}_{2}$ laser
Observation of parametric instabilities in the lower-hybria range of frequencies in the high-density tokamak

Y. Takase, M. Porkolab, J. J. Schuss,", R. L. Watterson, and C. L. Fiore

Plakase, M. Porkolab, J. J. Schuss," R. L. Watterson, and C. L. Fiore

R. E. Slusher and C. M. Surko

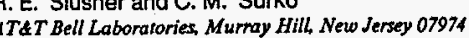

Received 24 July 1984; accepted 4 December 1984

Parametric decay processes have been studied using $\mathrm{rf}$ probes and $\mathrm{CO}_{2}$ laser scattering during the lower-hybrid wave heating and current drive experiments in the Alcator $C$ tokamak. The most important process is believed to be the nonresonant docay into ion-cyclotron quasimodes and/or of the injected pump wave and $\omega_{0}(0)$ is the lower-hybrid frequency evaluated at the plasma of the injected pump wave and $\omega_{\text {in }}(0)$ is absent or very weat. At higher densities $\left[\omega_{0} / \omega_{2}, 0 \mid \leqslant 2\right]$ strong parametric decay is observod absent or very weak. At higher deasiles $\left[\omega_{0} / \omega_{\mathrm{a}}(0) \leqslant 2\right]$ slrong parametric decay is observed which correlates well with ion tall formation near the plasma cdgen Above these densities the Parametric decay may be responsible, at least partially, for loss of wave power near the plasma periphery.

$f=4.6 \mathrm{GHz}$

I. INTRODUCTION

Most lower-hybrid wave heating and current drive experiments suffer degradation of efficiency at high-plasma densities such that $\omega_{0} / \omega_{11}(0) \leqslant 2$. At such densities parametric decay is of en observed and may be par thally responsible for the deterioradon of effeiencies. Here we present experiment resils on par ex probes and $\mathrm{CO}_{1}$ haser 25 ster for lin for lineaverage densides $n_{\text {, }} 21.5 \times 10^{14} \mathrm{~cm}^{-3}$ in hydrogen [comp cm in deuterium [Corresponding to $\left.\omega_{0} / a_{14}(0) \leq 2.2\right]$ at a kA. The excitation of parametric decay correlated well with the formation of ion tail near the plasma surface 43 Above these critical densities electron tails disappear and no signifcant heating or current drive have been observed. In this paper, we also compare these experimental observations with the theory of parametric decay instabilities. ${ }^{6-11}$ The un derstanding of parametric processes is important in applying lower-hybrid heating and current drive to high-density plas mas, where $\omega d \omega_{1 b}(0) \leqslant 2$.

The plan of the paper is as follows: In Sec. II theoretica estimates of growth rates and thresholds are presented. In Sec. III the results of ri probe measurements are presented and correlated with the formation of ion tail observed by the charge exchange analyzer. In Sec. IN the $\mathrm{CO}_{2}$ laser scattering data will be presented and compared with the $r$ probe data. In meretarion of the experimental results is also presenter.

"Present address: Raytheon Company, Wayland, Massachusects 01778
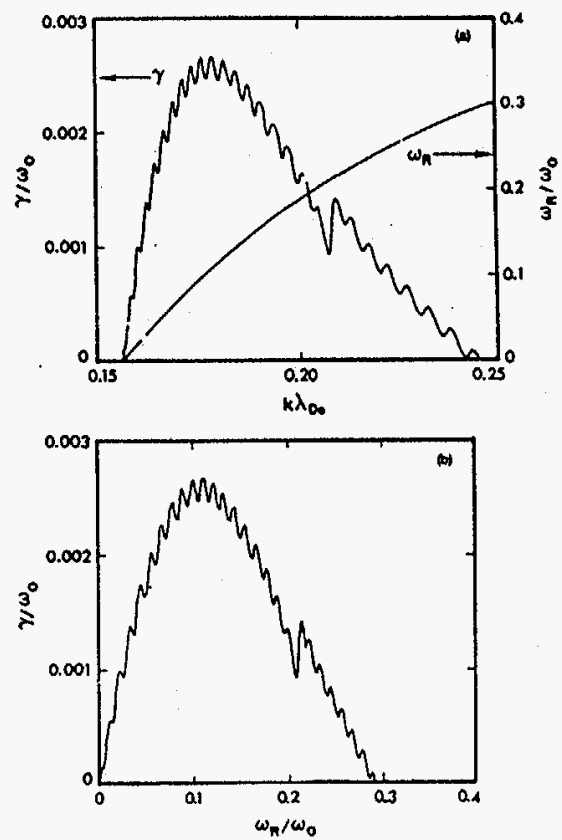

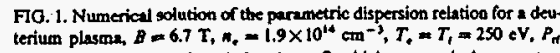
$=100 \mathrm{~kW}, c k_{0} / \omega_{0}=3$, and $c k_{1}-1 \omega_{t}=7$, which were typical parameters near tion of $\omega_{A}$. 

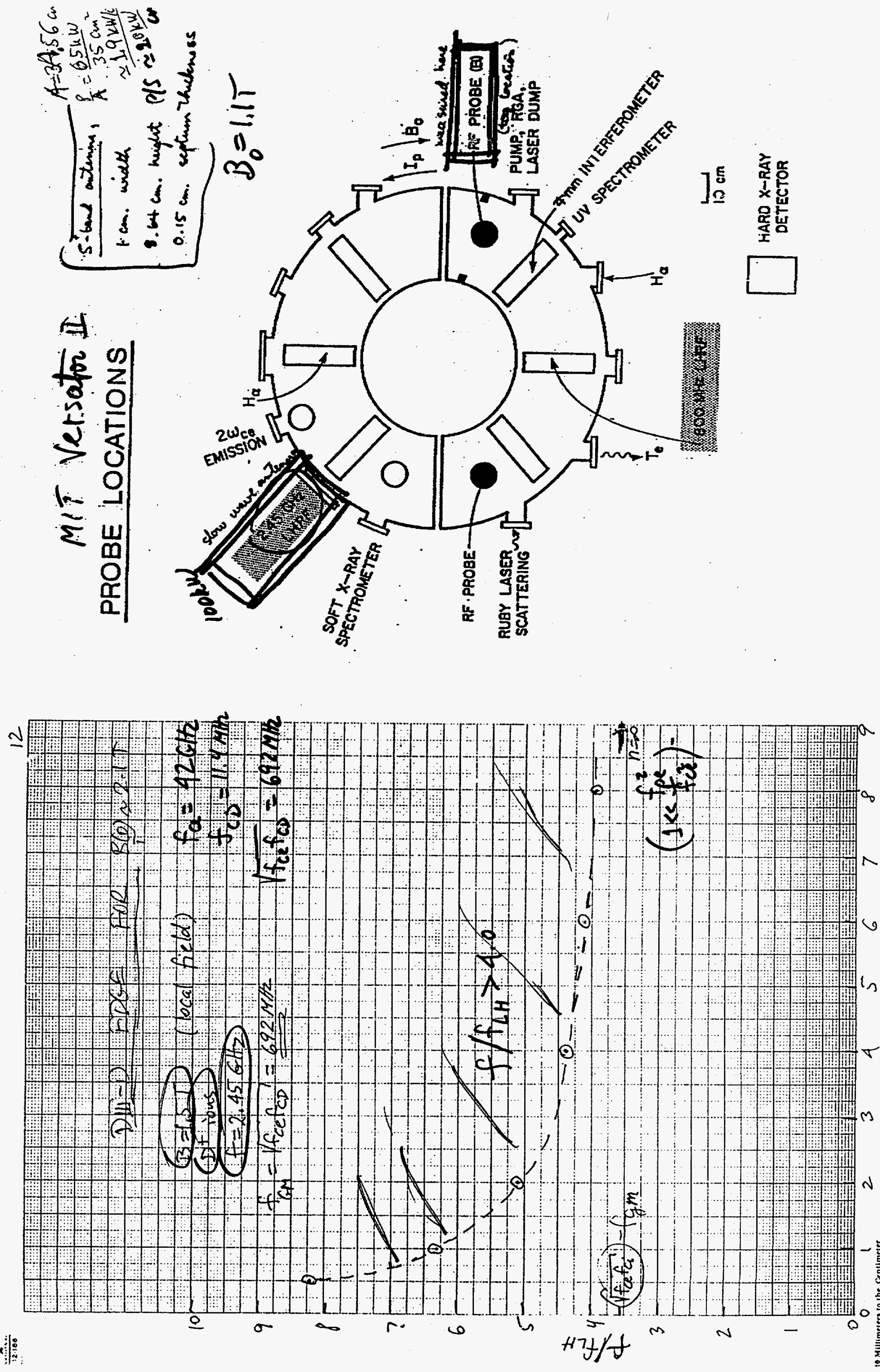


\section{Miklos PORKOLAB}

(510) $294-6401$

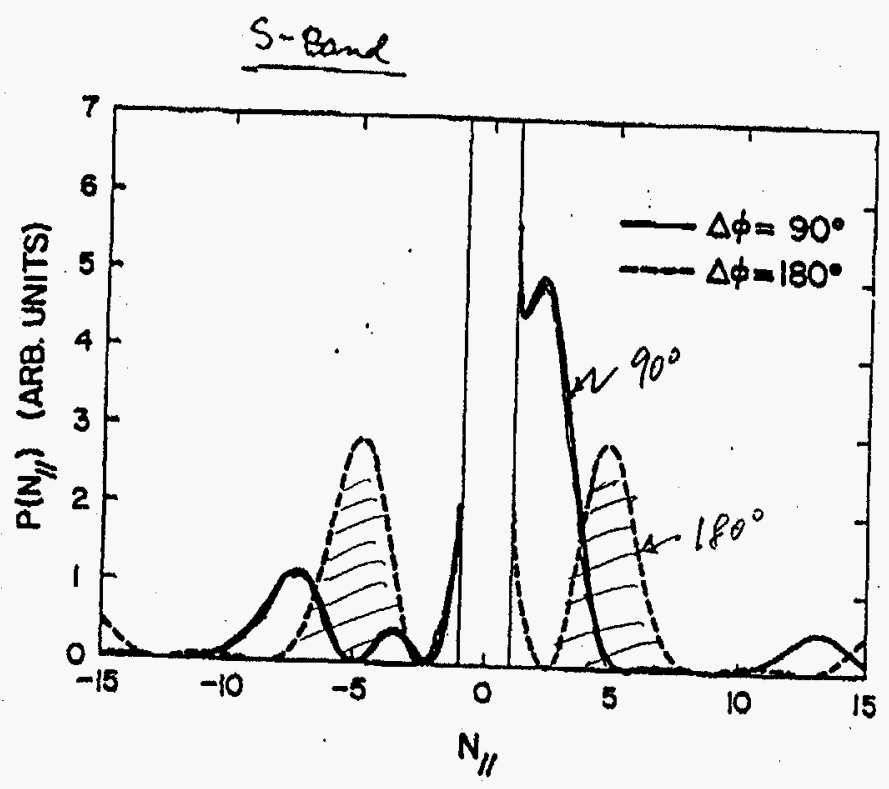

From: Joen VILASENOR

$\pi-281 \quad(617) 253-4174$

Fig. 3.5: Calculated $N_{\|}$power spectra of the S-band four-waveguide grill with $\Delta \phi=80^{\circ}, 180^{\circ}$ phasings, and plasma edge parameters $n_{i} / n_{\mathrm{c}}=10$ and
$L=n(d n / d x)^{-1}=1.2 \mathrm{~cm}$.

69
NUMU:R 8 MU PHYSICAL REVIEW LETTERS

19 August i985

while in Fig. 2(a), $/ / P$ vs $\bar{n}_{e}$ is shown. Both plots are consisient with the efficiency scaling of Fisch, with solid curves). All of the data here were obtained in $H_{2}$ solischarges, al an average toroidal field of $10-12 \mathrm{kG}$. and with $+90^{\circ}$ relative waveguide phasing. "Flat topping" is also possible, though (5-10)\% less efricient. with $+60^{\circ}$ and $+120^{\circ}$ phasings; other phases (includin $-90^{\circ}$ ) produce substantially less if current, as exrected, and to date flat topping of the toroidal plasma current has not been rossible.

The plasma hard $x$-ray emission has been measured rith a collimated 3-in. $x$ 3-in. Nal scintiliator detector tefore and during quasi-sieady-stale $2.45 .0 \mathrm{~Hz}$ current dive. The delector is localed below the tokamak, where it views the center of the plasma along a vertical thord aimed at a recessed top port. This configurabion, along with sufficient lead shielding, efrectively chiminates any background $x$-ray hux from the lokamak walls and limiters. As shown in Fig. 3, for curreni-drive discharges al $n_{0}-7 \times 10^{12} \mathrm{~cm}$. The levd of $x$-ray emission from the plasma is negligible in the injection of $85 \mathrm{~kW}$ of of power $\mathrm{Benerates}$ an enes the injection of $85 \mathrm{~kW}$ of 1 power relic electron ave specirum of $n_{1}-14$. The slope of the specirum $(-20 \mathrm{keV})$ remains nearly constant during the prasi-steady-state discharge. in addition to sustaining currents at constant levels with 2.45-GHz if, it has also been possible to ramp up plasma cursent, with negative loop voltage, at denivies $\bar{n}_{e} \leqslant 7 \times 10^{12} \mathrm{~cm}^{-3}$. Rampup rates as high as $100 \mathrm{kA} / \mathrm{s}$, for periods up io 15 nis (iimiled by the vert(al field system), have been recorded at a density of it the

1.7

value

3) in

igher-

higher

lan for

$s$ been

or the

t-drive

tharges
$\mathrm{cm}^{-3}$

rroduc

driven
iwes $P$. ergy 10 poloidal field energy was $\eta=0.06 \pm 0.02$, where $\eta \otimes \Delta\left(\frac{1}{2} L I^{2}\right) /\left(P_{s s} \Delta t\right)$, with $L=L_{\text {in }}+L_{\text {sus }}$ The uncertainty in the efficiency lies in the determination of the internal inductance from equilibrium measurements, since for these discharges $\beta_{0} \sim 1 / 2$. Nevertheless, this level of efficiency is consistent with the rampup theory of Fisch and Karney. el available to date $\left(P_{\text {net }} \leqslant 95 \mathrm{~kW}\right)$, if-current flat-top and rampup discharges have been restricted to densities at or below $\pi_{e}=1 \times 10^{13} \mathrm{~cm}^{-3}$. Al higher densities, with the $\mathrm{OH}$ primary open circuited, current drive is still observed as evidenced by reduced plasma
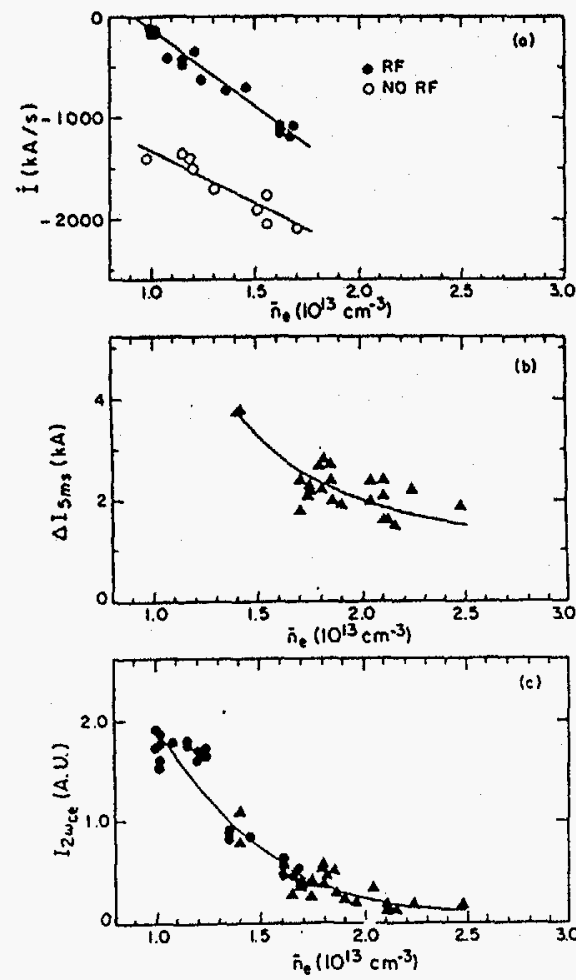

FiG. 4. (a) $d / / d t$ vs $\bar{n}_{e,}$ with and without if power (75-90 kW). for discharges with the $O H$ primary open circuited. (b) Incremental current increase $\Delta P(\mathrm{~s}$ ms into he ri pulse) clatron emission $l b_{2}$ us $\bar{n}_{0}$ for the discheres in (a) (circles) and (b) (uriangles).
Because of the relatively low $2.45 . \mathrm{GHz}$ if power lev. 


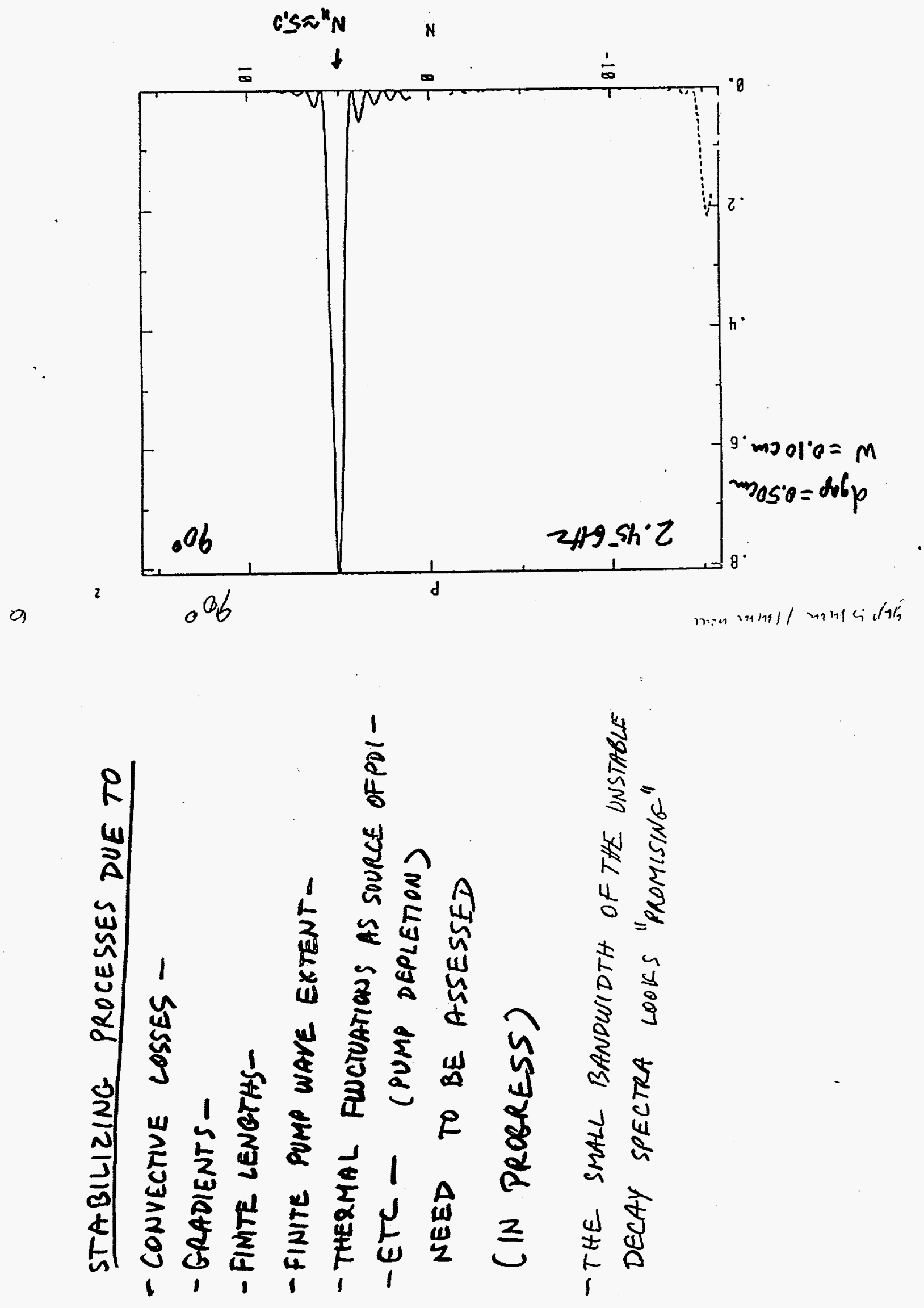
Gap $5 \mathrm{~mm} / 1 \mathrm{~mm}$ wall

$11<1)$

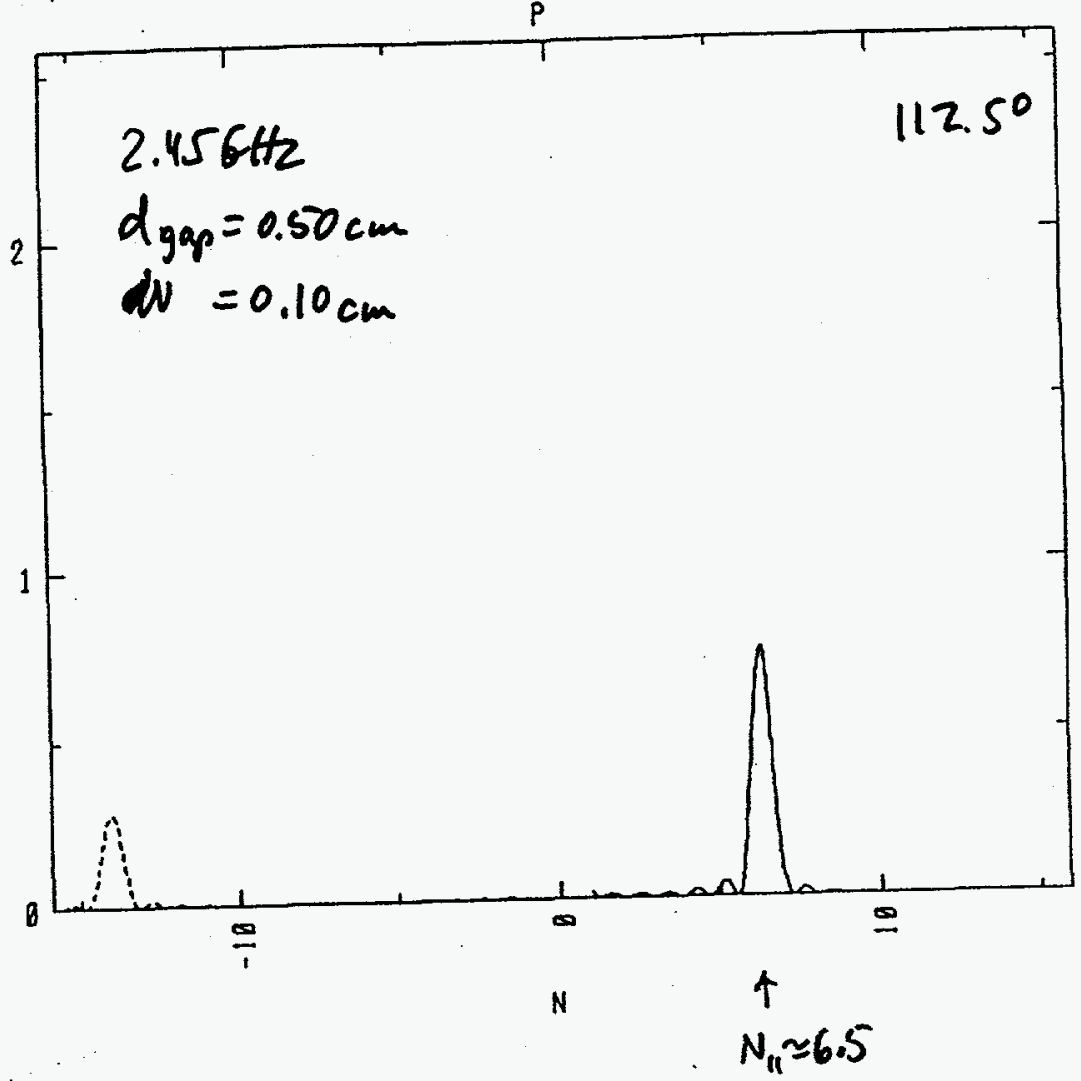

Fig. 2.4 b. Same as (a) but $\Delta \varphi=1125^{\circ}, y_{b}$

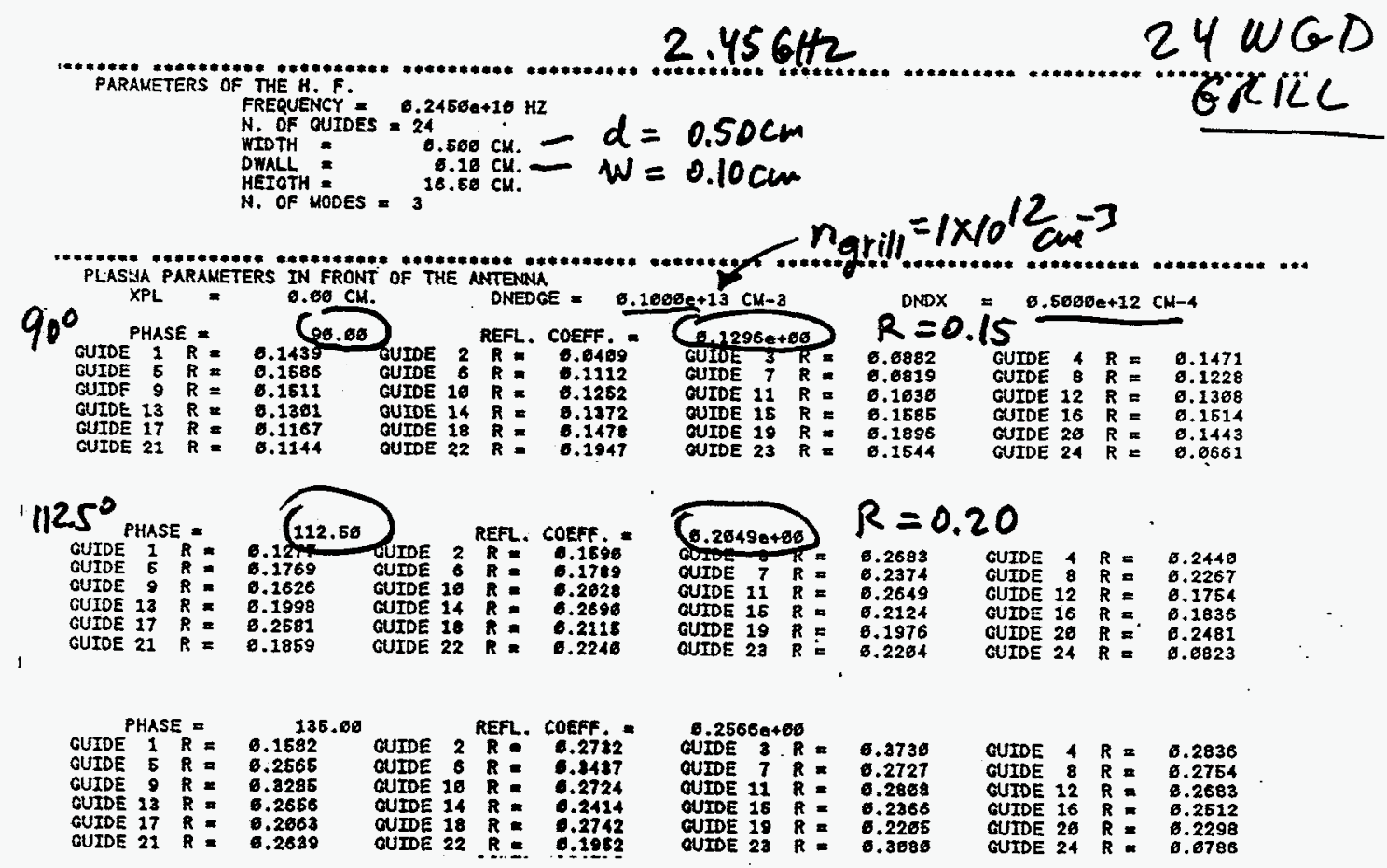


SUMMARY

1. FOR EDGE CURRENT PROFILE CONTROL. IN DIII-D, 2.45 GHz MAY BE a SUITABLE FREQUENCY

2. ACCESSIBILITY is Limiting waVe PENETRATION TO $T_{e}$ E 5 LeV; THIS D ACCEPTABLE FOR ALL FUTURE' SCENARIOS FOR EDGE CURRENT PROFILE CONTROL

3. THE LOWER FREQUENCY IS ADVANTAGEOUS FOR: THE LAUNCHER FABRICATION "EASE" (NEW LAUNCHER WITH HIE NIL IS NEEDED; ? SHOULD CONSIDER NOVEL LAUNCHER CONCEPT)

4. THE BIG UNCERTAINTY IS LACK OF DATA ON THE CD-DENSITY LIMIT IN THE "LARGE DIELECTRIC CONSTANT" REGIs (LE, $\left.\omega_{p e}^{2} / \omega_{C P}^{2} \sim 3-6\right)$

- THEORETICALLY WE EXPECT NO MAJOR OBSTICLES TO ELECTRON INTERACTION SINCE $\omega / \omega L{ }_{L H} \sim 4.0$; NEVERTHELESS, THE POTENTIAL ROLE OF DI MUST BE STUDIED IN MORE DETAIL; THIS IS IN PROGRESS 5. Further EXPERIMENTS ON VERSATOR COMD BE CARRIED OUT (NOT FUNDED AT PRESENT) - SIMLARLY, IT -60 COULD LOOK AT PDI AT LOW Bo wITH $2 \mathrm{GHL}$ 


\title{
Appendix I \\ Frequency, Density Limits and Parametric Decay Instabilities
}

\author{
M E M O R N D U M
}

DATE: January 25, 1993

TO: $\quad$ K. I. Thomassen

S. L. Allen

FROM: $\quad$ M. Porkolab

M. E. Fenstermacher

SUBJECT: $\quad 2.45 \mathrm{GHz}$ LHCD for Edge Control in DIII-D: An Assessment Summary of Findings at LLNL on 1/12-15/93

We have re-examined the question of LHCD (Lower-Hybrid Current-Drive) applications in DIII-D in view of the latest TPX DIII-D physics developments and needs.

It appears that the greatest interest in LHCD on DIII-D may be in the area of counter-current-drive $(C C D)$ in the region of $(r / a) \simeq 0.85-1.0$ or $\Psi \simeq 0.80-1.0$. The potential uses for CCD with LHCD include:

(a) Cancellation of the excessive amounts of edge bootstrap current during the VH-mode phase. This would allow potential stabilization of the edge-kink modes ( $n=2-5)$ and an extension of the VH-mode to steadystate. We see this as one of the most critical issues facing in the DIII-D Program, with major benefits to TPX where steady-state VH-mode is highly desirable.

(b) If the advanced divertor program in DIII-D is successful and the density gradients near the edge will be more benign and do not lead to excessive bootstrap currents, there is still a need for edge CCD as shown by recent MHD studies in TPX (see work of Ramos and Zakharov). In this case a net negative edge current distribution may be possible with a subsequent increase in the value of $g_{\text {Troyon }}$ for $\mathrm{q}_{\mathrm{O}} \geq 2$, achievement of 
high bootstrap currents $\left(f_{\mathrm{bs}} \geq 0.7\right)$, high beta and second stability. For low edge densities (ie. peaked density and/or pressure profiles), the power necessary to drive LHCD CCD would be less than in the case of VH-modes in past year's experiments (where the densities often peak near the edge, i.e., at $\mathrm{r} / \mathrm{a} \sim 0.8-0.9$ ).

(c) CCD near the edge would help to increase $l_{i}$ and result in improved confinement.

(d) Edge current-drive is not possible in DIII-D with either fast waves (FWCD) or electron cyclotron waves (ECCD) because of lack of wave absorption (FWCD) or because of ultra-low current-drive efficiency due to trapping (ECCD). Hence, LHCD is complementary to other currentdrive techniques presently envisaged within the DIII-D program plan.

We have previously discussed the potential application of LHCD on DIII-D for current-drive profile control, in particular for the achievement of 2nd stability. This requires co-LHCD about half way in radially. This proposal was not favorably received by General Atomics physicists for the following reasons: given the low B-field of DIII-D, it would have been necessary to keep the plasma parameters to relatively low values $\left.\mathrm{n}_{\mathrm{e}}(0) \lesssim 5 \times 10^{19}, \mathrm{~T}_{\mathrm{e}} \leq 5 \mathrm{keV}\right)$. This is a consequence of poor accessibility $\left(\mathrm{N}_{\|} \geq 3\right.$ at $\mathrm{B} \simeq 2.1 \mathrm{~T}, \mathrm{n}_{\mathrm{e}}(0) \simeq 5 \times 10^{19} \mathrm{~m}^{-3}$ ) which then limits penetration to $\mathrm{T} \backslash \mathrm{S}(, \mathrm{e}) \backslash \mathrm{O}(>, \sim) 5 \mathrm{keV}$ (strong quasi-linear absorption access at $\mathrm{T}_{\mathrm{e}} \geq 50 / \mathrm{N}_{\|}^{2}$ ). There is a serious question whether the plasma parameters can be kept at such a low value during the "all out" $\mathrm{I}_{\mathrm{P}}=2 \mathrm{MA}, 5 \%$ beta, $10 \mathrm{sec}$ target scenario. Therefore, we have re-examined the potential use of LHCD on DII-D and arrived at the new scenario of edge counter LHCD.

To arrive at a reasonable cost factor, we have re-examined the frequency that might be acceptable (and perhaps even desirable from considerations of the launcher fabrication).

Our present thinking is that a $2.45 \mathrm{GHz}$ system might be acceptable from a physics point-of-view. This would then lead to significant cost savings since the 3 MW LHCD system presently at Garching may be usable. Specifically, we realize that in the edge region $B \lesssim 1.5$ Tesla, and at high densities the lower hybrid frequency is not the ion plasma frequency but the geometric-mean frequency $f_{L H} \simeq \sqrt{f_{C_{i}} f_{c e}}$, which in deuterium at $B \sim 1.5$ Tesla is about $600 \mathrm{MHz}$, and hence $\mathrm{f} / \mathrm{f}_{\mathrm{LHZ}} 4$ everywhere in the plasma periphery. In the attached figure we show a simple plot of $f / f_{\mathrm{LH}}$ versus density at $B=1.5 \mathrm{~T}$. Thus, we may be 
above the "density-limit" which usually occurs once $f / f_{L H} \leq 2$ and parametric decay instabilities produce ion tails and absorb the waves. Therefore, it appears that $2.45 \mathrm{GHz}$ may be an acceptable frequency (and in fact desirable) even at $\mathrm{n} \lesssim$ $2 \times 10^{19} \mathrm{~m}^{-3}$ ), if PDI can be mitigated. Unfortunately, most ot the data in past experiments has been obtained for $\omega_{p e}^{2} / \omega_{c e}^{2}<1$, and in that case $f_{L H}$ goes to $f_{p i}$. However, in DIII-D we would be in the regime $f_{p e}^{2} / f_{c e}^{2}>1$ (at high denisties) and little (if any) data exists here on the density limit. The only LHCD tokamak experiment in this regime may have been on Versator II (Ref. 3), where current drive effects have been noted at $\mathrm{f}=2.45 \mathrm{GHz}, \mathrm{B}_{\mathrm{O}}=1.0$ Tesla, $\mathrm{n} \lesssim 2 \times 10^{19} \mathrm{~m}^{-3}$ even in hydrogen. Nevertheless, some parametric instability phenomenon have been observed. However, these experiments were performed in hydrogen, in which case $\mathrm{f}_{\mathrm{LH}}$ is closer to the pump frequency than in deuterium, and hence more prone to PDI. Although Versator II is presently shut down due to lack of funding, the machine is on a "stand-by" basis and is fully operational. The following equipment is on the machine and is mostly operational.

1. Plasma parameters in hydrogen are Ip $\leqslant 50 \mathrm{kA}, \mathrm{n}_{\mathrm{eo}} \leqslant 5 \times 10^{19} \mathrm{~m}^{-3}, \mathrm{~T} \mathrm{e} \lesssim 400$ $\mathrm{eV}, \mathrm{T}_{\mathrm{eo}} \lesssim 150 \mathrm{eV}, \mathrm{a} \simeq 13 \mathrm{~cm}, \mathrm{R}_{\mathrm{o}}=40 \mathrm{~cm}$, which are characteristic of the DIII$\mathrm{D}$ edge region. Tokamak operation in $\mathrm{He}_{4}$ (or $\mathrm{D}_{2}$ ) could carried out. Operating parameters should be similar (or better) than in hydrogen but are not documented.

2. Two LH systems are available, in particular a $100 \mathrm{~kW}, 2.45 \mathrm{GHz}$ system is operational, including a 4 waveguide grill with $\mathrm{N} \| \sim 2$ (current-drive phasing) or $\mathrm{N}_{\|} \sim 5(0, \pi, 0, \pi)$ phasing. A new modified grill could easily be fabricated yielding $N_{\|} \sim 4-6$ with co-(or counter-) current-drive phasing. Relevant data on PDI at DIII-D edge parameters could be investigated within a 12 month time frame at a reasonable cost. Available diagnostics (in working order) include:

(a) 7 channel $\mathrm{NaI}$ hard $\mathrm{x}$-ray detector array for PHA of energetic electron $(\varepsilon \gtrsim 50 \mathrm{keV})$

(b) Radially scanable SiLi detector for soft $x$-rays $(\varepsilon \lesssim 50 \mathrm{keV})$

(c) Multiple RF probe arrays to pick up PDI spectra

(d) Retarding potential edge energy analyzer probe to measure $10 \mathrm{eV}-1$ $\mathrm{keV}$ electron and ion distribution 
(e) Charge exchange analyzer to look at fast ions

(f) Microwave interferometers $(2 \mathrm{~mm}, 4 \mathrm{~mm})$ to measure density profiles

(g) $2 \omega_{\text {ce }}$ superheterodyne receiver to look at non-thermal emission

(h) MHD loops

(i) Spectrum analyzer to look at RF probe spectra.

The RF power density injected in Versator II $\left(2 \mathrm{~kW} / \mathrm{cm}^{2}\right)$ is similar to that envisaged for DIII-D

\section{Initial PDI growth rate studies}

We have begun studying parametric decay instability (PDI) growth rates and substantial progress has been made with the Porkolab PDI code at LLNL. We have examined four regions characteristic of the plasma edge:

(a) $\mathrm{n}_{\mathrm{e}}=5 \times 10^{19} \mathrm{~m}^{-3}, \mathrm{~T}_{\mathrm{e}}=500 \mathrm{eV}, \mathrm{T}_{\mathrm{i}}=500 \mathrm{eV}$

(b) $\mathrm{n}_{\mathrm{e}}=2 \times 10^{19} \mathrm{~m}^{-3}, \mathrm{~T}_{\mathrm{e}}=100 \mathrm{eV}, \mathrm{T}_{\mathrm{i}}=100 \mathrm{eV}, 50 \mathrm{eV}, 200 \mathrm{eV}, 500 \mathrm{eV}$

(c) $\mathrm{n}_{\mathrm{e}}=1 \times 10^{19} \mathrm{~m}^{-3}, \mathrm{~T}_{\mathrm{e}}=50 \mathrm{eV}, \mathrm{T}_{\mathrm{i}}=50 \mathrm{eV}, 200 \mathrm{eV}$

(d) $\mathrm{n}_{\mathrm{e}}=5 \times 10^{18} \mathrm{~m}^{-3}, \mathrm{~T}_{\mathrm{e}}=10 \mathrm{eV}, \mathrm{T}_{\mathrm{i}}=10 \mathrm{eV}$

Significant growth rates have been obtained in some of the cases especially in the low density region very near the launcher. The growth rates decrease further inward toward the high density and high temperature region.

Typical maximum growth rates obtained are given in the Table of Growth Rates. We see that as the temperature (and the density) increase, high up on the VHmode gradient $\left(\mathrm{n}_{\mathrm{e}}=5 \times 10^{19} \mathrm{~m}^{-3}, \mathrm{~T}_{\mathrm{e}}=500 \mathrm{eV}\right)$, the maximum growth rate at 2.0 $\mathrm{kW} / \mathrm{cm}^{2}$ injected power level is of the order of $\gamma / \omega_{0} \sim 0.002$ (an exact value was not yet obtained; rather, we scale from the $4.0 \mathrm{~kW} / \mathrm{cm}^{2}$ level where $\gamma / \omega_{O}$ $\sim 0.0038$ ). These values are relatively modest as we shall see when we determine the convective threshold. A few $\mathrm{cm}$ farther out toward the edge where $\mathrm{n}_{\mathrm{e}}=2 \mathrm{x}$ $10^{19} \mathrm{~m}^{-3}, \mathrm{~T}_{\mathrm{e}}=100 \mathrm{eV}$, we obtain $\gamma / \omega_{\mathrm{O}} \sim 0.03$, a rather substantial value. It should be noted that this is at a frequency $\omega / \omega_{\mathrm{O}} \sim 0.05$, or $\omega / \Omega_{\mathrm{D}} \sim 12$, a rather high harmonic ion cyclotron quasi-mode. Other harmonics should also be unstable but with lower growth rates. The sidebands are propagating lower hybrid waves. We see that even farther out toward the edge $\left(\mathrm{n}_{\mathrm{e}} \leqslant 1 \times 10^{19} \mathrm{~m}^{-3}, \mathrm{~T}_{\mathrm{e}} \lesssim 30\right.$ 
$\mathrm{eV})$, the growth rates are comparable. The effects of convective losses must be evaluated for more thorough assessment of the danger of pump depletion. Again, small scale experiments in a device such as Versator II would be invaluable.

\section{Convective losses and PDI threshold conditions.}

As seen earlier, for the present parameters $\omega_{0} / \omega_{\mathrm{LH}} \gtrsim 4$, and hence to a good approximation the lower-hybrid wave dispersion relationship is given by the cold plasma limit, namely:

$$
\omega=\omega_{L H}\left(1+\frac{k_{\|}^{2}}{k^{2}} \frac{m_{i}}{m_{e}}\right)^{1 / 2} \approx \omega_{L H} \frac{k_{1 H}}{k} \sqrt{\frac{m_{i}}{m_{e}}}=\omega_{L H} \frac{k_{\| 1}}{k}
$$

where $k^{2}=k_{1}^{2}+k_{\perp}^{2} \approx k_{\perp}^{2}$ and where

$$
\varpi_{L H}=\omega_{L H} \sqrt{\frac{m_{i}}{m_{e}}}=\frac{\omega_{p i}\left(m_{i} / m_{e}\right)^{1 / 2}}{\left(1+\omega_{p e}^{2} / \omega_{c e}^{2}\right)^{1 / 2}}=\frac{\omega_{p e}}{\left(1+\omega_{p e}^{2} / \omega_{c e}^{2}\right)^{1 / 2}}
$$

As we saw earlier, we take $B \cong 1.5 T$ near the edge, and therefore $\left(f_{\mathrm{ce}} \mathrm{f}_{\mathrm{CD}}\right)^{1 / 2}=692$ MHz. For densities $\mathrm{n}_{\mathrm{e}} \gtrsim 1 \times 10^{18} \mathrm{~m}^{-3}, k^{2} \cong k_{\perp}^{2}, \quad k_{\| 1}^{2} \ll k_{\perp}^{2}$, and the group velocities are given by

$$
\begin{gathered}
v_{g \perp}=\frac{\partial \omega}{\partial k_{\perp}}=\frac{-\omega}{k_{\perp}}=\frac{-c \omega}{N_{\|} \varpi_{L H}} \\
v_{g \|}=\frac{\partial \omega}{\partial k \|}=\frac{\varpi_{L H}}{k_{\|}}=\frac{c \omega}{k_{\|} c}=\frac{c}{N_{\|}}
\end{gathered}
$$

The maximum growth rate occurs for $\underline{E_{o}} \times \underline{B_{o}}$ coupling rather than for Ello coupling (Ref. 4, Eq. 5) if

$$
\frac{k_{\|}^{-}}{k_{\perp}^{-}}<\left(\frac{\omega_{o}}{\omega_{c e}}\right)^{1 / 2}=0.24
$$

or

$$
\frac{\omega^{-}}{\omega_{L H}^{-}} \cong\left(\frac{\omega_{o}}{\omega_{L H} \sqrt{m_{i} / m_{e}}}\right)^{1 / 2}<0.24
$$

We see that at densities $n_{\mathrm{e}} \gtrsim 5 \times 10^{18} \mathrm{~m}^{-3}, \omega_{2} / \varpi_{L H} \leq 0.1$, and in particular, at $\mathrm{n}_{\mathrm{e}} \gtrsim$ $2 \times 10^{19} \mathrm{~m}^{-3}, \omega_{2} / \varpi_{L H} \leq 0.083$. Thus, E $\left.\right|_{\|}$coupling becomes comparable to $\underline{E_{o}} \times \underline{B_{o}}$ 
coupling only at densities $\mathrm{n}_{\mathrm{e}} \lesssim 1 \times 10^{18} \mathrm{~m}^{-3}$. Therefore, the relevant convective thresholds are 4,5 :

(a) $\frac{\gamma L_{y}}{\left|v_{y}^{-}\right|} \cong \frac{\omega_{L H} \gamma L_{y} N_{\|}^{-}}{c \omega^{-}} \geq \pi \quad$ (loss in the poloidal direction)

and

(b) $\frac{\gamma L_{z}}{\left|v_{z}^{-}\right|} \cong \frac{\gamma L_{z} N_{\|}^{-}}{c} \geq \pi \quad$ (loss in the toroidal direction)

Taking a grill with height $\mathrm{Ly}=40 \mathrm{~cm}$ and width $\mathrm{L}_{z}=30 \mathrm{~cm}$ (48 waveguides toroidally, $N_{\| o} \cong 6, \Delta \phi=\pi / 2$, and 4 rows vertically, each $\sim 10 \mathrm{~cm}$ high), the above conditions become,

(a) $\left(\frac{\gamma}{\omega_{o}}\right) \frac{\omega_{o} N_{\|}^{-} L_{y}}{c \pi}\left(\frac{\omega_{L H}}{\omega_{2}}\right) \geq 1 \quad$ (poloidal loss )

and

(b) $\left(\frac{\gamma}{\omega_{o}}\right) \frac{\omega_{o} N_{\|}^{-} L_{z}}{c \pi} \geq 1$ (toroidal loss)

where (b) will be the more restrictive condition since $\mathrm{L}_{\mathrm{y}} \sim \mathrm{L}_{z}$ and $\bar{\sigma}_{L H} / \omega_{2}>1$ (namely, convective losses in the toroidal direction dominate). Now let us consider different edge regions. Using $N_{\|}^{-}=8, L_{z} \cong 30 \mathrm{~cm}, n_{e}=1 \times 10^{19} \mathrm{~m}^{-3}$, condition (b) gives $\gamma / \omega_{0} \gtrsim 0.025$ (and similar results are obtained for $n_{e}=$ $5 \times 10^{18} \mathrm{~m}^{-3}, \mathrm{~N}_{\|} \cong 10$ ) so that the threshold condition is marginnaly satisfied for $\mathrm{P} / \mathrm{A}=2 \mathrm{~kW} / \mathrm{cm}^{2}, \mathrm{~T}_{\mathrm{e}}=\mathrm{T}_{\mathrm{i}}$. However, for $\mathrm{T}_{\mathrm{i}} / \mathrm{T}_{\mathrm{e}} \geq 4$, as observed in DIII-D experiments, $\gamma / \omega_{0} \leq 0.013$ for $\mathrm{P} / \mathrm{A} \lesssim 2 \mathrm{~kW} / \mathrm{cm}^{2}$ and the threshold condition is not met. In addition, even if this threshold condition is met, past experiments indicate no serious pump depletion under similar circumstances. In particular, in this regime $\omega_{p e}^{2} / \omega_{c e}^{2}<<1$, as in all other experiments. The new regime ( $\left.\omega_{p e}^{2} / \omega_{c e}^{2} \geq 3\right)$ is restricted to high densities further inside the plasma where $n_{e} \gtrsim$ $5 \times 10^{19} \mathrm{~m}^{-3}$. We see that in this regime $\mathrm{N}_{\|} \cong 2, \mathrm{~T}_{\mathrm{i}}>\mathrm{T}_{\mathrm{e}}$ and $\mathrm{T}_{\mathrm{e}} \gtrsim 500 \mathrm{eV}$, and $\gamma / \omega_{0} \leq 0.004$. Thus, condition (b) requires a growth rate of $\gamma / \omega_{0} \simeq 0.10$, and we are nowhere near threshold for PDI. In an intermediate regime where $\mathrm{n}_{\mathrm{e}}=2 \times 10^{19} \mathrm{~m}^{-3}, \mathrm{~T}_{\mathrm{e}} \cong 100 \mathrm{eV}, \mathrm{T}_{\mathrm{i}} / \mathrm{T}_{\mathrm{e}} \sim(2-4), \mathrm{N}_{\|}^{-}=8$, we have a growth rate of $\gamma / \omega_{\mathrm{o}}$ $\sim 0.016-0.011$ (depending on ion temperature) whereas the required minimum threshold is again $\gamma / \omega_{0} \cong 0.025$. Thus, we do not expect serious pump depletion effects, or prevention of wave penetration.

\section{Conclusions from the PDI Studies}


The conditions for convective thresholds (ie. an $\exp (2 \pi)$ growth of the decay waves) can not be met at edge conditions $n_{e}>1 \times 10^{19} \mathrm{~m}^{-3}, T_{i} \simeq 4 T_{e}, T_{e} \simeq 50 \mathrm{eV}$. Thus, only within a few centimeters in front of the grill mouth, where $n_{e} \lesssim$ $5 \times 10^{18} \mathrm{~m}^{-3}$, and $\mathrm{T}_{\mathrm{e}} \sim 10 \mathrm{eV}, \mathrm{T}_{\mathrm{e}}=\mathrm{T}_{\mathrm{i}}$ could the threshold be met for a grill of $\mathrm{L}_{\mathrm{z}} \gtrsim 30$ $\mathrm{cm}$ toroidal extent. In this regime $\omega_{p e}^{2} / \omega_{c e}^{2} \ll 1, \omega_{\mathrm{o}} / \omega_{\mathrm{LH}} \gtrsim 8$. As in previous experiments, we do not expect serious problems with pump wave penetration. Further if $T_{i} \cong 4 T_{e}$, the threshold is not met. Nevertheless, we recommend that the toroidal extent of the grill not exceed $\mathrm{L}_{\mathrm{z}} \sim 50 \mathrm{~cm}$.

\section{Some comments on long pulse issues:}

We recognize that the ASDEX 3.0 MW, $2.45 \mathrm{GHz}$ system is rated at $1.0 \mathrm{sec}$ pulse operation at full-power mostly limited by the circulators (rated for full-reflected power at $0.5 \mathrm{MW}$ each). However, if we were to consider fabrication of a new, high $\mathrm{N}_{\|}$grill of the multi-junction type, then we could reduce the reflected power to at most $10 \%$ of the incident power. Under such conditions the $3.7 \mathrm{GHz}$ Thompson tubes do not require circulators (and TORE-SUPRA does not use any!). For the Varian $2.45 \mathrm{GHz}$ tubes, complete circulator protection would be available for a $10 \mathrm{sec}$ pulse length (at $300 \mathrm{~kW}$ total reflected power). The Varian tubes are more prone to damage at high reflected power. Thus, it appears that for the cost of a new grill (of the type we might actually want!) which should not cost above $1 \mathrm{M} \$$, a long-pulse $2.45 \mathrm{GHz}$ system may become available. 


\section{Table of Growth Rates}

Typical frequencies, $\omega / \omega_{\mathrm{O}^{\prime}}$, and maximum growth-rates, $\gamma / \omega_{\mathrm{O}^{\prime}}$ at three locations in the edge region are as follows:

For $\mathrm{n}_{\mathrm{e}}=5.0 \times 10^{19} \mathrm{~m}^{-3}, \mathrm{~T}_{\mathrm{e}}=500 \mathrm{eV}, \mathrm{B}=1.5 \mathrm{~T}$

\begin{tabular}{|lllllllll|}
\hline $\begin{array}{l}P / A \\
\left(\mathrm{~kW} / \mathrm{cm}^{2}\right)\end{array}$ & $\mathrm{T}_{\mathrm{e}} / \mathrm{T}_{\mathrm{i}}$ & $\omega / \omega_{\mathrm{O}}$ & $\omega^{-} / \omega_{\mathrm{O}}$ & $\begin{array}{l}\gamma / \omega_{\mathrm{O}} \\
(\max .)\end{array}$ & $k \lambda_{\mathrm{De}}$ & $\mathrm{N}_{\|_{\mathrm{O}}}$ & $\mathrm{N}_{\|}$ & $\mathrm{N}_{\|}{ }^{-}$ \\
\hline 8.0 & 1.0 & 0.058 & 0.942 & 0.007 & 0.0807 & 4.0 & 6.0 & 2.0 \\
4.0 & 1.0 & 0.058 & 0.942 & 0.0038 & 0.0807 & 4.0 & 6.0 & 2.0 \\
2.0 & 1.0 & 0.058 & 0.942 & 0.0020 & 0.0807 & 4.0 & 6.0 & 2.0 \\
20.0 & 1.0 & 0.060 & 0.940 & 0.014 & 0.0808 & 4.0 & 6.0 & 2.0 \\
\hline
\end{tabular}

[Note: growth rates are rather small at $\mathrm{P} / \mathrm{A}<4.0 \mathrm{~kW} / \mathrm{cm}^{2}$

For $\mathrm{n}_{\mathrm{e}}=2 \times 10^{19} \mathrm{~m}^{-3}, \mathrm{~T}_{\mathrm{e}}=100 \mathrm{eV}, \mathrm{B}=1.5 \mathrm{~T}$

\begin{tabular}{|lllllllll|}
\hline $\begin{array}{l}\mathrm{P} / \mathrm{A} \\
\left(\mathrm{kW} / \mathrm{cm}^{2}\right)\end{array}$ & $\mathrm{T}_{\mathrm{e}} / \mathrm{T}_{\mathrm{i}}$ & $\omega / \omega_{\mathrm{O}}$ & $\omega^{-} / \omega_{\mathrm{O}}$ & $\gamma / \omega_{\mathrm{O}}$ & $\mathrm{k} \lambda_{\mathrm{De}}$ & $\mathrm{N}_{\|_{\mathrm{O}}}$ & $\mathrm{N} \|$ & $\mathrm{N}_{\|}{ }^{-1}$ \\
\hline 2.0 & 1.0 & 0.040 & 0.960 & 0.0175 & 0.090 & 6.0 & 12.0 & 6.0 \\
1.0 & 1.0 & 0.041 & 0.959 & 0.0110 & 0.090 & 6.0 & 12.0 & 6.0 \\
2.0 & 1.0 & 0.037 & 0.965 & 0.0120 & 0.0754 & 6.0 & 10.0 & 4.0 \\
2.0 & 1.0 & 0.038 & 0.967 & 0.0154 & 0.0765 & 4.0 & 10.0 & 6.0 \\
1.0 & 1.0 & 0.037 & 0.963 & 0.0097 & 0.077 & 4.0 & 10.0 & 6.0 \\
& & & & & & & & \\
4.0 & 1.0 & 0.055 & 0.945 & 0.033 & 0.1085 & 6.0 & 14.0 & 8.0 \\
2.0 & 1.0 & 0.055 & 0.945 & 0.021 & 0.1085 & 6.0 & 14.0 & 8.0 \\
1.0 & 1.0 & 0.049 & 0.951 & 0.013 & 0.108 & 6.0 & 14.0 & 8.0 \\
0.5 & 1.0 & 0.048 & 0.952 & 0.008 & 0.108 & 6.0 & 14.0 & 8.0 \\
0.25 & 1.0 & 0.047 & 0.953 & 0.005 & 0.108 & 6.0 & 14.0 & 8.0 \\
0.125 & 1.0 & 0.047 & 0.953 & 0.002 & 0.108 & 6.0 & 14.0 & 8.0 \\
& & & & & & & & \\
2.0 & 2.0 & 0.049 & 0.951 & 0.025 & 0.108 & 6.0 & 14.0 & 8.0 \\
2.0 & 0.5 & 0.053 & 0.947 & 0.016 & 0.110 & 6.0 & 14.0 & 8.0 \\
2.0 & 0.25 & 0.072 & 0.928 & 0.011 & 0.108 & 6.0 & 14.0 & 8.0 \\
\hline
\end{tabular}


For $\mathrm{n}_{\mathrm{e}}=1 \times 10^{19} \mathrm{~m}^{-3}, \mathrm{~T}_{\mathrm{e}}=50 \mathrm{eV}, \mathrm{B}=1.5 \mathrm{~T}$

\begin{tabular}{|lcccccccc|}
\hline $\begin{array}{l}\mathrm{P} / \mathrm{A} \\
\left(\mathrm{kW} / \mathrm{cm}^{2}\right)\end{array}$ & $\mathrm{T}_{\mathrm{e}} / \mathrm{T}_{\mathrm{i}}$ & $\omega / \omega_{\mathrm{O}}$ & $\omega^{-} / \omega_{\mathrm{O}}$ & $\gamma / \omega_{\mathrm{O}}$ & $\mathrm{k}^{\mathrm{De}}$ & $\mathrm{N}_{\|_{\mathrm{O}}}$ & $\mathrm{N}_{\|}$ & $\mathrm{N}_{\|^{-}}$ \\
\hline 4.0 & 1.0 & 0.034 & 0.966 & 0.029 & 0.085 & 6.0 & 14.0 & 8.0 \\
2.0 & 1.0 & 0.031 & 0.969 & 0.020 & 0.085 & 6.0 & 14.0 & 8.0 \\
1.0 & 1.0 & 0.029 & 0.971 & 0.014 & 0.085 & 6.0 & 14.0 & 8.0 \\
0.5 & 1.0 & 0.028 & 0.972 & 0.009 & 0.085 & 6.0 & 14.0 & 8.0 \\
0.25 & 1.0 & 0.027 & 0.973 & 0.006 & 0.085 & 6.0 & 14.0 & 8.0 \\
0.125 & 1.0 & 0.027 & 0.973 & 0.003 & 0.085 & 6.0 & 14.0 & 8.0 \\
& & & & & & & & \\
2.0 & 0.25 & 0.034 & 0.966 & 0.013 & 0.085 & 6.0 & 14.0 & 8.0 \\
1.0 & 0.25 & 0.031 & 0.969 & 0.0076 & 0.085 & 6.0 & 14.0 & 8.0 \\
\hline
\end{tabular}

For $\mathrm{n}_{\mathrm{e}}=5 \times 10^{18} \mathrm{~m}^{-3}, \mathrm{~T}_{\mathrm{e}}=10 \mathrm{eV}, \mathrm{B}=1.5 \mathrm{~T}$

\begin{tabular}{|lcccccccc|}
\hline $\begin{array}{l}\mathrm{P} / \mathrm{A} \\
\left(\mathrm{kW} / \mathrm{cm}^{2}\right)\end{array}$ & $\mathrm{T}_{\mathrm{e}} / \mathrm{T}_{\mathrm{i}}$ & $\omega / \omega_{\mathrm{o}}$ & $\omega^{-} / \omega_{\mathrm{o}}$ & $\begin{array}{l}\gamma / \omega_{\mathrm{o}} \\
(\max .)\end{array}$ & $\mathrm{k} \lambda$ De & $\mathrm{N}_{\|_{\mathrm{o}}}$ & $\mathrm{N}_{\|}$ & $\mathrm{N}_{\|^{-}}$ \\
\hline 8.0 & 1.0 & 0.033 & 0.967 & 0.033 & 0.048 & 6.0 & 16.0 & 10.0 \\
4.0 & 1.0 & 0.029 & 0.971 & 0.024 & 0.048 & 6.0 & 16.0 & 10.0 \\
\hline
\end{tabular}




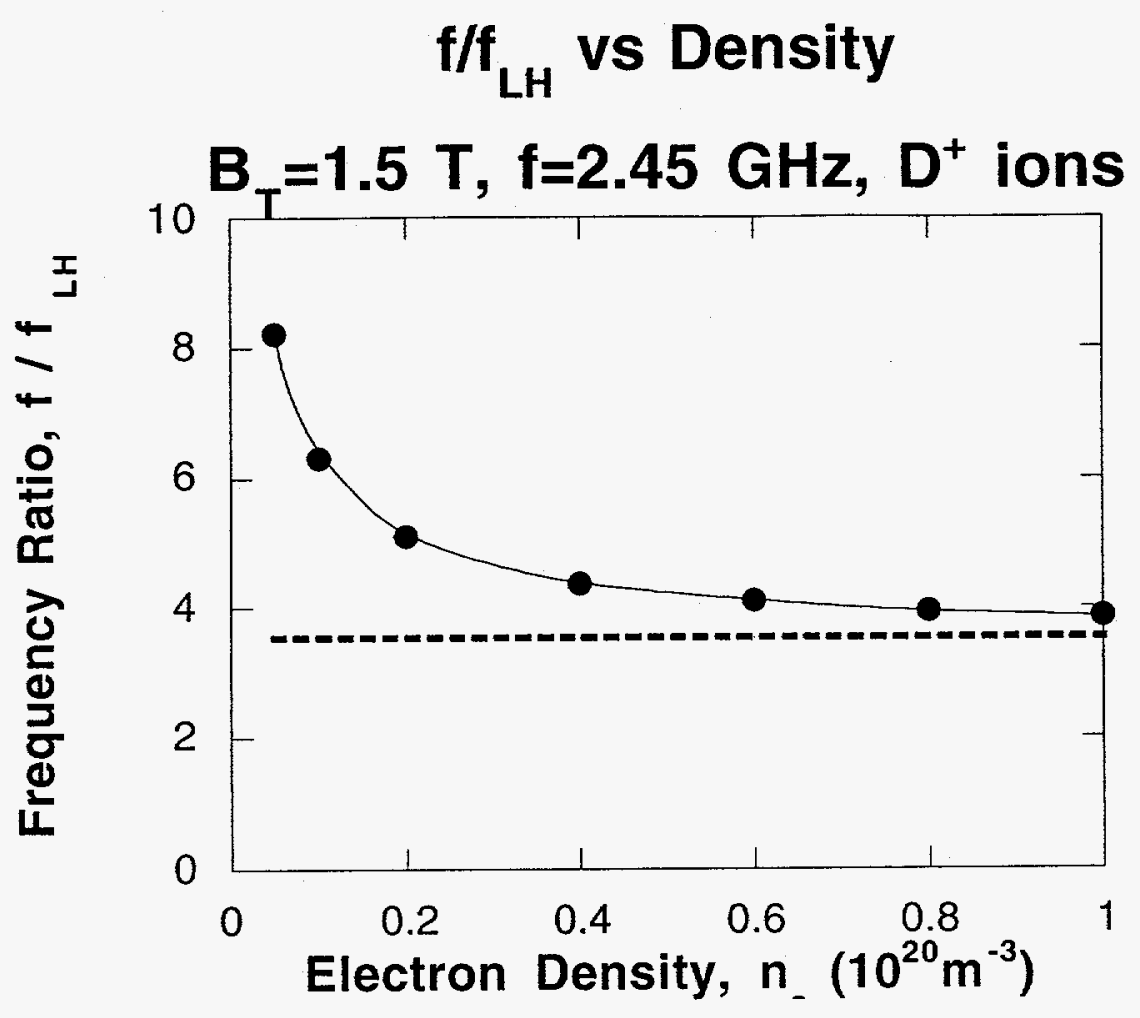

